\title{
Clinical analysis of the "small plateau" sign on the flow-volume curve followed by deep learning automated recognition
}

Yimin Wang ${ }^{1 \dagger}$, Wenya Chen ${ }^{1 \dagger}$, Yicong Li ${ }^{2,3 \dagger}$, Changzheng Zhang ${ }^{3}$, Lijuan Liang ${ }^{1}$, Ruibo Huang ${ }^{1}$, Jianling Liang ${ }^{1}$, Yi GaO ${ }^{1 * \dagger}$ and Jinping Zheng ${ }^{1 * \dagger}$

\begin{abstract}
Background: Small plateau (SP) on the flow-volume curve was found in parts of patients with suspected asthma or upper airway abnormalities, but it lacks clear scientific proof. Therefore, we aimed to characterize its clinical features.

Methods: We involved patients by reviewing the bronchoprovocation test (BPT) and bronchodilator test (BDT) completed between October 2017 and October 2020 to assess the characteristics of the sign. Patients who underwent laryngoscopy were assigned to perform spirometry to analyze the relationship of the sign and upper airway abnormalities. SP-Network was developed to recognition of the sign using flow-volume curves.

Results: Of 13,661 BPTs and 8,168 BDTs completed, we labeled 2,123 (15.5\%) and 219 (2.7\%) patients with the sign, respectively. Among them, there were 1,782 (83.9\%) with the negative-BPT and 194 (88.6\%) with the negative-BDT. Patients with SP sign had higher median FVC and FEV $1 \%$ predicted (both $P<.0001$ ). Of 48 patients (16 with and 32 without the sign) who performed laryngoscopy and spirometry, the rate of laryngoscopy-diagnosis upper airway abnormalities in patients with the sign (63\%) was higher than those without the sign (31\%) $(P=0.038)$. SP-Network achieved an accuracy of $95.2 \%$ in the task of automatic recognition of the sign.

Conclusions: SP sign is featured on the flow-volume curve and recognized by the SP-Network model. Patients with the sign are less likely to have airway hyperresponsiveness, automatic visualizing of this sign is helpful for primary care centers where BPT cannot available.
\end{abstract}

Keywords: Airway responsiveness, Deep learning, Flow-volume curve, Pulmonary function test, Small plateau sign

*Correspondence: misstall2@163.com; jpzhenggy@163.com

†Yimin Wang, Wenya Chen and Yicong Li contributed equally to this work

as co-first authors

${ }^{\dagger} \mathrm{Yi}$ Gao and Jinping Zheng contributed equally to this work as cocorresponding author

${ }^{1}$ National Center for Respiratory Medicine, State Key Laboratory of Respiratory Disease, National Clinical Research Center for Respiratory Disease, Guangzhou Institute of Respiratory Health, First Affiliated Hospital of Guangzhou Medical University, Yanjiang Road 151, Guangzhou 510120, Guangdong, People's Republic of China Full list of author information is available at the end of the article

\section{Background}

Spirometry flow-volume curve displays airflow versus volume during maximum forced inspiration and expiration, it can be influenced by various factors [1]. According to international standards, types of ventilatory defects can be inferred from the configuration of the curve, obstructive abnormalities are thought to be a concave shape on the curve, the curve of a restrictive ventilatory defect shows a convex pattern [2]. Furthermore, Miller and Hyatt [3] found that flattening on the inspiratory and/or expiratory phase of the curve could suggest upper airway obstruction. Flow oscillations referred to as 
a "saw-tooth" sign seen on curves are thought to be an indicator of obstructive sleep apnea [4]. The flow-volume curve display is diversified in patients with different diseases, as the disease progresses, so does the curve configuration.

Li et al. [5] first found a small plateau (SP) in the early phase of expiratory flow (mostly located at the phase from PEF to $\left.\mathrm{FEF}_{50 \%}\right)$. They identified the sign in 228 of 808 patients (28.2\%) who completed bronchoprovocation test (BPT), about 196 (86\%) of patients had negative$\mathrm{BPT}$ indicating that patients with the sign maybe have a negative trend prevalence of airway hyperresponsiveness (AHR). Moreover, they found that patients with the sign had a prevalence of symptoms due to upper airway abnormalities, including severe cough and hoarseness when complete the methacholine challenge maneuvers, which indicated that the sign may be related to abnormal structure or function of the upper airway. Xie et al. [6]
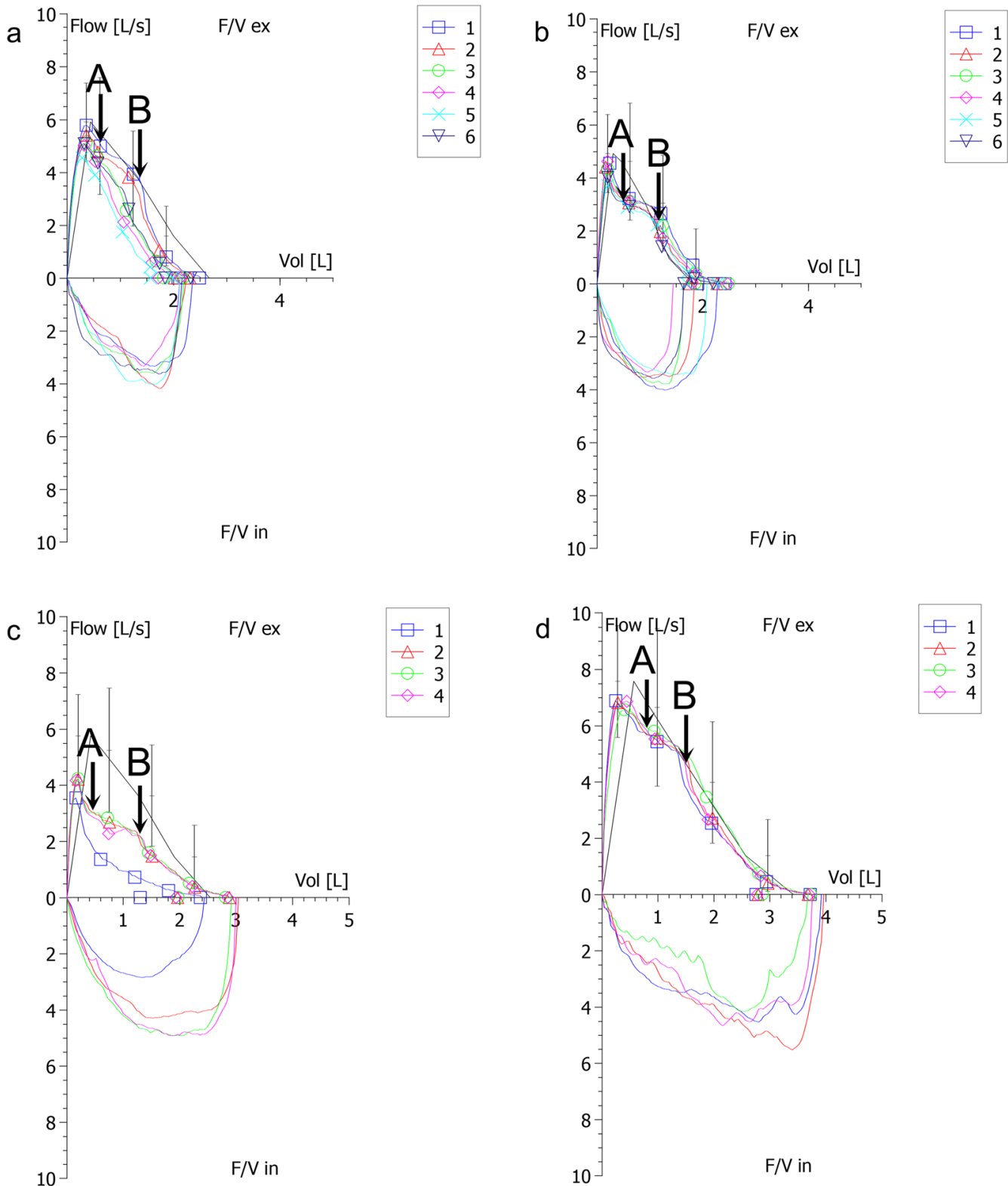

Fig. 1 Representative examples of the SP sign in BPTs and BDTs. a SP sign in positive BPT. Curve $1=$ pre-BPT, SP sign (+); Curve $5=$ post-BPT, SP $(-)$, b SP sign in negative BPT. Curve $1=$ pre-BPT, SP sign (+); Curve $6=$ post-BPT, SP sign (+). c SP sign in positive BDT; Curve 1=pre-BDT, SP sign ( -$)$; Curve $4=$ post-BDT, SP $(+)$. $\mathbf{d}$ SP sign in negative BDT; Curve $1=$ pre-BDT, SP sign $(+)$; Curve $4=$ post-BDT, SP sign (+). Point $\mathrm{A}=$ the start point of the SP sign; Point $\mathrm{B}=$ the end point of the SP sign. $\mathrm{SP}=$ small plateau; $\mathrm{BPTs}=$ bronchoprovocation tests; $\mathrm{BDT}=$ bronchodilator tests 


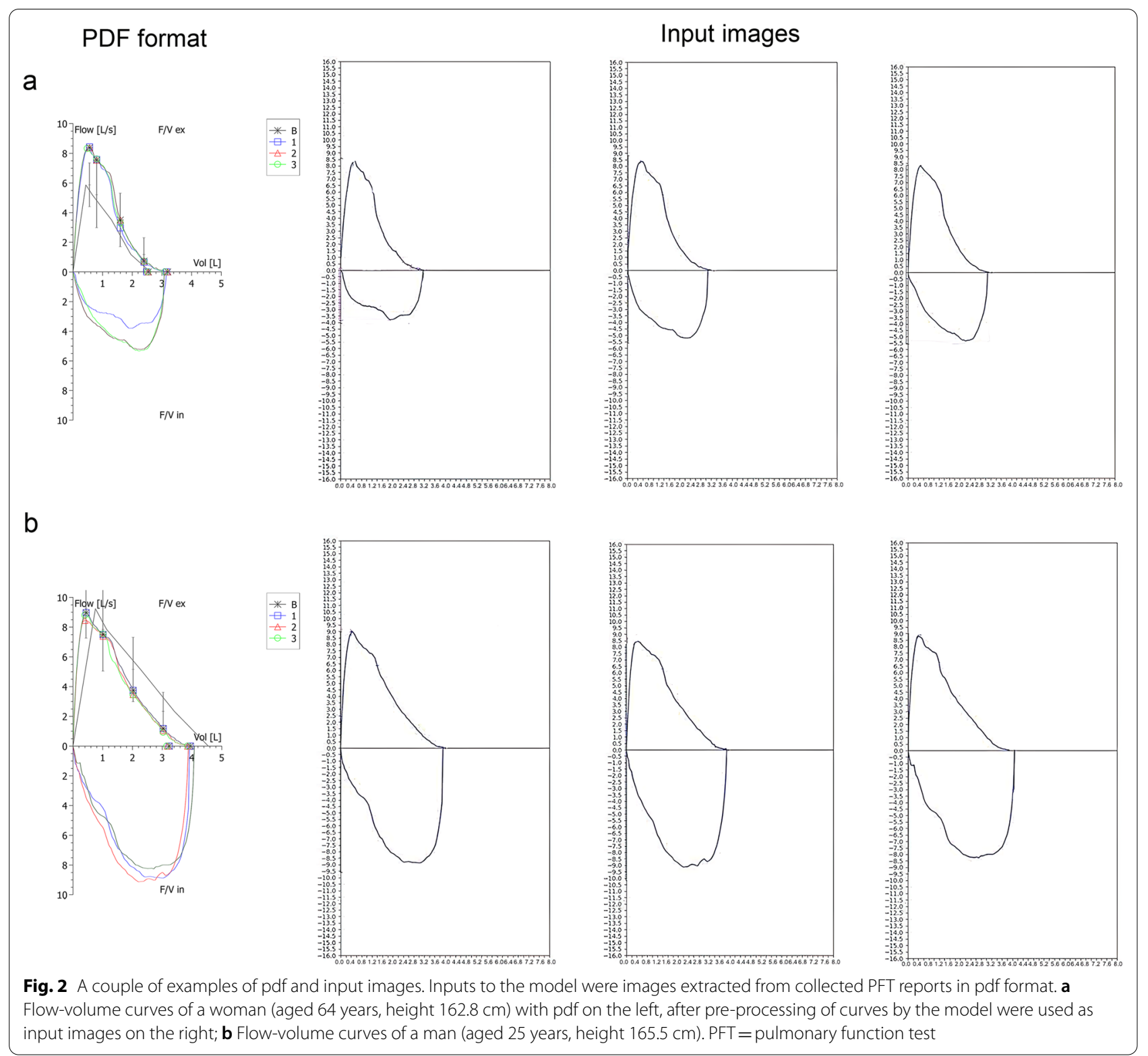

similarly found that patients were more often negativeBPT (95.2\%) in a total of 124 subjects with SP sign who performed BPT, they further confirmed that patients with SP sign may less likely to have AHR. BPT is used to evaluate AHR which is most commonly associated with asthma [7]. However, due to a variety of reasons, BPT cannot be available in primary care settings. In addition, the test is time-consuming and usually requires specialists. At present, asthma has a rapid rise in prevalence in low-income and middle-income countries [8]. In highincome countries, it has high rates of exacerbation and re-admissions [9], which has been proved associated with lung function [10]. Some previous studies have focused on finding simple methods to evaluate AHR and diagnose asthma, like questionnaires and baseline spirometry parameters [11, 12]. The identification and application of the SP sign may have crucial value in predicting the absence of AHR to avoid unnecessary testing.

With the availability of portable spirometers in primary care, forced spirometry (FS) has become easily accessible to all levels of healthcare. In clinical practice, however, most non-professionals lack the ability or experience to identify the configuration of the flow-volume curve, which may lead to the need for a simple and effective approach for visual inspection of the curve. The application of artificial intelligence in medicine is rising quickly 


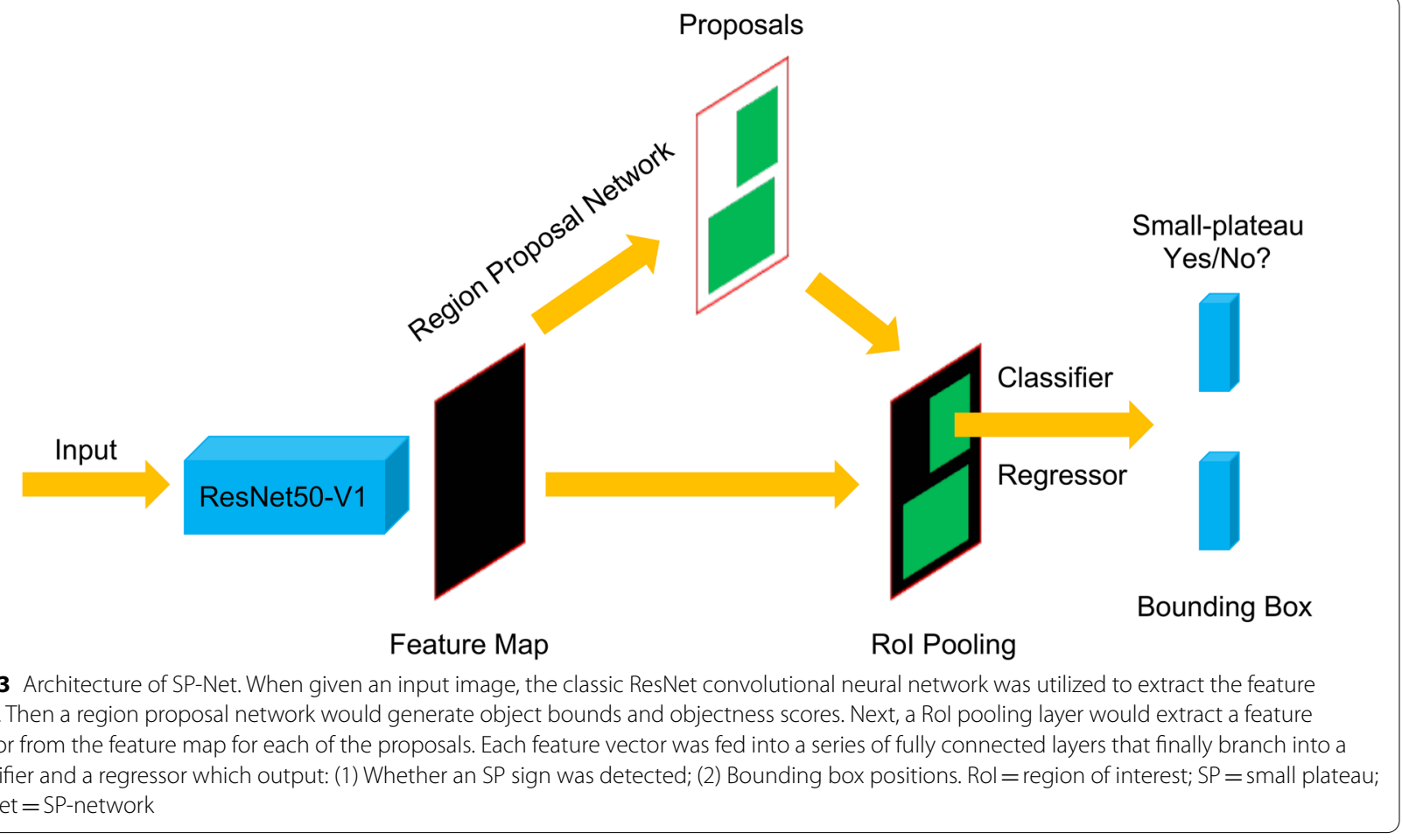

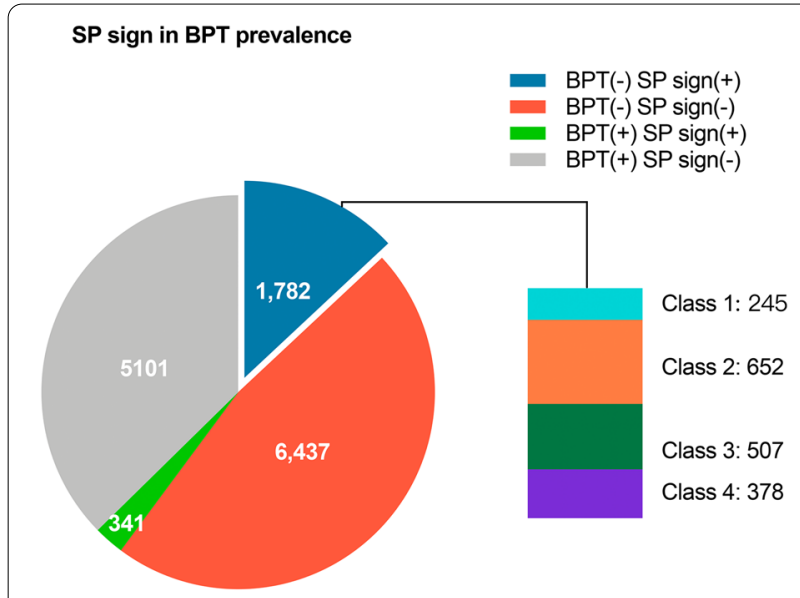

Fig. 4 Prevalence of SP sign of all patients in BPTs. BPT (-) SP sign $(+)$ was defined as patients with SP sign and had negative-BPTs; BPT (-) SP sign (-) was defined as patients without SP sign and had negative-BPTs; BPT (+) SP sign (+) was defined as patients with SP sign and had positive-BPTs; BPT (+) SP sign (-) was defined as patients without SP sign and had positive-BPTs. Classes were defined as follows: class $1=($ Vol A - Vol B $) \times 100 / F V C$ ratio $\leq 10 \%$ in $\mathrm{BPT}(-)$; class $2=(\mathrm{Vol} \mathrm{A}-\mathrm{Vol} \mathrm{B}) \times 100 / \mathrm{FVC}$ ratio $>10 \%$ to $\leq 20 \%$ in $\mathrm{BPT}(-)$; class $3=(\mathrm{Vol} \mathrm{A}-\mathrm{Vol} \mathrm{B}) \times 100 / \mathrm{FVC}$ ratio $>20 \%$ to $\leq 30 \%$ in $\mathrm{BPT}(-)$; class $4=($ Vol A - Vol B) $\times 100 / F V C$ ratio $>30 \%$ in BPT $(-)$. $\mathrm{BPT} s=$ bronchoprovocation tests; $\mathrm{Vol}=$ volume; $\mathrm{SP}=$ small plateau

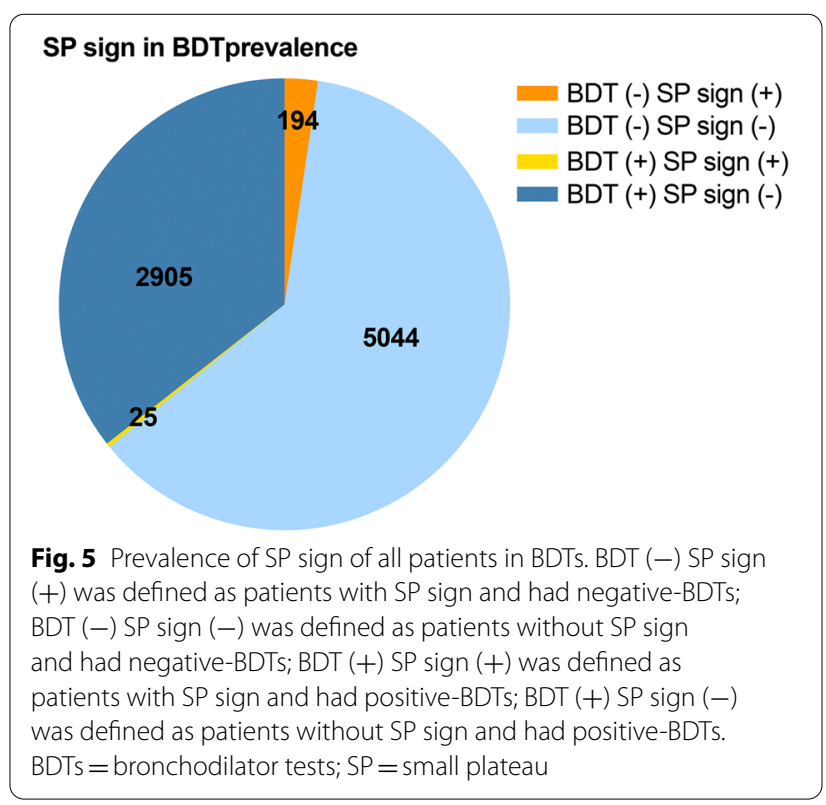

[13]. Deep learning is a branch of artificial intelligence that could provide a simple and useful tool to automate spirogram recognition [14]. Though it is possible that some simpler computer vision $(\mathrm{CV})$ techniques could also solve the problem, deep learning-based CV methods are able to outperform those traditional $\mathrm{CV}$ and signal processing methods in most scenarios [15-19]. 
Table 1 Characteristic parameters

\begin{tabular}{|c|c|c|c|c|c|c|}
\hline \multirow[t]{2}{*}{ Parameter } & \multicolumn{6}{|l|}{ SP sign } \\
\hline & $\begin{array}{l}\text { BPT }(-) \\
(\mathrm{N}=1782)\end{array}$ & $\begin{array}{l}\text { BPT }(+) \\
(\mathrm{N}=341)\end{array}$ & $P$ value & $\begin{array}{l}\text { BDT (-) } \\
(\mathrm{N}=194)\end{array}$ & $\begin{array}{l}\text { BDT }(+) \\
(\mathrm{N}=25)\end{array}$ & $P$ value \\
\hline \multicolumn{7}{|l|}{ Pre-BDT or BPT, \% } \\
\hline Vol A/FVC ratio & $\begin{array}{l}19.0 \\
(16.1,23.1)\end{array}$ & $\begin{array}{l}19.6 \\
(15.9,23.2)\end{array}$ & .468 & $\begin{array}{l}17.5 \\
(13.9,21.8)\end{array}$ & $\begin{array}{l}0.0 \\
(0.0,9.8)\end{array}$ & $<.0001$ \\
\hline Flow A/PEF ratio & $\begin{array}{l}90.6 \\
(86.2,93.8)\end{array}$ & $\begin{array}{l}87.6 \\
(83.6,92.1)\end{array}$ & $<.0001$ & $\begin{array}{l}88.6 \\
(82.5,93.1)\end{array}$ & $\begin{array}{l}0.0 \\
(0.0,78.0)\end{array}$ & $<.0001$ \\
\hline Vol B/FVC ratio & $\begin{array}{l}40.9 \\
(32.5,49.1)\end{array}$ & $\begin{array}{l}37.6 \\
(31.4,44.5)\end{array}$ & $<.0001$ & $\begin{array}{l}30.2 \\
(22.9,36.8)\end{array}$ & $\begin{array}{l}0.0 \\
(0.0,14.3)\end{array}$ & $<.0001$ \\
\hline Flow B/PEF ratio & $\begin{array}{l}73.7 \\
(64.9,81.3)\end{array}$ & $\begin{array}{l}73.5 \\
(67.8,79.9)\end{array}$ & .404 & $\begin{array}{l}78.3 \\
(69.6,85.2)\end{array}$ & $\begin{array}{l}0.0 \\
(0.0,71.1)\end{array}$ & $<.0001$ \\
\hline$($ Vol A - Vol B)/FVC ratio & $\begin{array}{l}19.9 \\
(13.2,28.4)\end{array}$ & $\begin{array}{l}16.6 \\
(11.0,22.7)\end{array}$ & $<.0001$ & $\begin{array}{l}10.0 \\
(6.3,16.6)\end{array}$ & $\begin{array}{l}0.0 \\
(0.0,3.3)\end{array}$ & $<.0001$ \\
\hline (Flow A - Flow B)/PEF ratio & $\begin{array}{l}15.9 \\
(10.1,22.3)\end{array}$ & $\begin{array}{l}12.4 \\
(8.3,18.1)\end{array}$ & $<.0001$ & $\begin{array}{l}8.7 \\
(5.5,13.8)\end{array}$ & $\begin{array}{l}0.0 \\
(0.0,3.7)\end{array}$ & $<.0001$ \\
\hline \multicolumn{7}{|l|}{ Post-BDT or BPT, \% } \\
\hline Vol A/FVC ratio & $\begin{array}{l}18.6 \\
(15.2,23.0)\end{array}$ & $\begin{array}{l}0.0 \\
(0.0,10.2)\end{array}$ & $<.0001$ & $\begin{array}{l}17.5 \\
(14.9,22.1)\end{array}$ & $\begin{array}{l}16.6 \\
(14.9,19.1)\end{array}$ & .083 \\
\hline Flow A/PEF ratio & $\begin{array}{l}90.3 \\
(85.2,94.1)\end{array}$ & $\begin{array}{l}0.0 \\
(0.0,68.7)\end{array}$ & $<.0001$ & $\begin{array}{l}89.2 \\
(83.8,93.4)\end{array}$ & $\begin{array}{l}88.0 \\
(83.9,93.3)\end{array}$ & .498 \\
\hline Vol B/FVC ratio & $\begin{array}{l}35.0 \\
(26.6,43.9)\end{array}$ & $\begin{array}{l}0.0 \\
(0.0,15.6)\end{array}$ & $<.0001$ & $\begin{array}{l}33.8 \\
(25.4,40.3)\end{array}$ & $\begin{array}{l}32.9 \\
(25.2,37.9)\end{array}$ & .331 \\
\hline Flow B/PEF ratio & $\begin{array}{l}76.5 \\
(66.4,84.2)\end{array}$ & $\begin{array}{l}0.0 \\
(0.0,56.9)\end{array}$ & $<.0001$ & $\begin{array}{l}76.2 \\
(68.2,83.0)\end{array}$ & $\begin{array}{l}73.5 \\
(69.2,80.0)\end{array}$ & .258 \\
\hline (Vol A - Vol B)/FVC ratio & $\begin{array}{l}14.1 \\
(8.8,22.4)\end{array}$ & $\begin{array}{l}0.0 \\
(0.0,3.6)\end{array}$ & $<.0001$ & $\begin{array}{l}12.8 \\
(8.5,20.6)\end{array}$ & $\begin{array}{l}15.8 \\
(10.3,20.2)\end{array}$ & .226 \\
\hline (Flow A - Flow B)/PEF ratio & $\begin{array}{l}11.7 \\
(6.8,18.2)\end{array}$ & $\begin{array}{l}0.0 \\
(0.0,2.5)\end{array}$ & $<.0001$ & $\begin{array}{l}11.5 \\
(7.0,16.8)\end{array}$ & $\begin{array}{l}13.2 \\
(8.7,19.0)\end{array}$ & .181 \\
\hline
\end{tabular}

Data are presented as median values and quartiles unless otherwise noted. Statistical comparisons based on percent unless otherwise noted for characteristic parameters of small plateau sign specific values. $P$ values $<.05$ were considered to represent a significant difference in prevalence between $S P$ signs of the negative and positive BPT or BDT categories

BPT, bronchoprovocation test; BDT, bronchodilator test; SP, small plateau; Vol, volume; FVC, forced vital capacity; PEF, peak expiratory flow

The reason behind this is that the deep learning model often encompasses a large parameter space and does not require hand-crafted features but instead automatically learns appropriate features. These characteristics give the deep learning model a stronger capacity to model the task and better generalization abilities compared to traditional methods. Since deep learning has produced promising results in tackling the display of the curve, we hypothesize that the application of deep learning is ideal for the automatic recognition of the SP sign.

In trying to analyze the potential use of the SP sign as a negative marker for AHR in patients with suspected asthma, we retrospectively reviewed the characteristics of the SP sign in BPT. Since $\mathrm{FEV}_{1}<60 \%$ predicted is a contraindication for BPT, we also reviewed bronchodilator tests (BDTs) to involve patients with severe ventilatory defects. Furthermore, for patients who required laryngoscopy according to specialists' decision, FS was performed after they have completed the laryngoscopy tests to assess the laryngoscopy-diagnosis upper airway abnormalities of patients with the sign. At last, we leveraged deep learning algorithms to automatically tackle the recognition problem of the SP sign.

\section{Methods}

This study was conducted at the First Affiliated Hospital of Guangzhou Medical University, using BPTs and BDTs data from October 2017 to October 2020 and FS data during the period 8 March 2021 to 26 March 2021. The study was approved by the Ethics Committee of the First Affiliated Hospital of Guangzhou Medical University (approval NO.: 2020124).

\section{Pulmonary function tests (PFTs)}

BPTs and BDTs were performed on MasterScreenPneumo (Jaeger, Germany) and QuarkPFT (COSMED, Italy). FS tests were performed by portable spirometer with U-BREATH PF680 (E-linkcare, China). Quality control of pre-and post- PFTs followed by guidelines, baseline spirometry should achieve three acceptable $\mathrm{FEV}_{1}$ 
Table 2 PFT values of patients with pre-BPTs

\begin{tabular}{|c|c|c|c|c|c|c|}
\hline \multirow[t]{2}{*}{ Parameter } & \multicolumn{3}{|l|}{$\mathrm{BPT}(+)$} & \multicolumn{3}{|l|}{ BPT (-) } \\
\hline & $\begin{array}{l}\text { SP sign }(+) \\
(N=341)\end{array}$ & $\begin{array}{l}S P \text { sign }(-) \\
(N=5101)\end{array}$ & $P$ value & $\begin{array}{l}S P \text { sign }(+) \\
(N=1782)\end{array}$ & $\begin{array}{l}S P \text { sign }(-) \\
(N=6437)\end{array}$ & $P$ value \\
\hline Age, years & $37(24,52)$ & $21(8,48)$ & $<.0001$ & $39(30,51)$ & $40(30,53)$ & .173 \\
\hline Sex, n (\%) & & & $<.0001$ & & & $<.0001$ \\
\hline Male & $121(35 \%)$ & $2618(51 \%)$ & & $714(40 \%)$ & $3162(49 \%)$ & \\
\hline Female & $220(65 \%)$ & 2483 (49\%) & & 1068 (60\%) & 3275 (51\%) & \\
\hline $\mathrm{BMI}, \mathrm{kg} / \mathrm{m}^{2}$ & $\begin{array}{l}22.3 \\
(19.6,24.9)\end{array}$ & $\begin{array}{l}20.1 \\
(16.0,23.6)\end{array}$ & $<.0001$ & $\begin{array}{l}22.6 \\
(20.4,24.8)\end{array}$ & $\begin{array}{l}22.6 \\
(20.1,25.0)\end{array}$ & .337 \\
\hline $\begin{array}{l}\text { Obesity, } n(\%) \\
\left(\mathrm{BMI} \geq 30 \mathrm{~kg} / \mathrm{m}^{2}\right)\end{array}$ & $13 / 341(4 \%)$ & $114 / 5101(2 \%)$ & .062 & $54 / 1782(3 \%)$ & $90 / 6437$ (1\%) & $<.0001$ \\
\hline FVC \% predicted, \% & $\begin{array}{l}102.4 \\
(94.9,112.0)\end{array}$ & $\begin{array}{l}98.8 \\
(90.9,107.3)\end{array}$ & $<.0001$ & $\begin{array}{l}103.0 \\
(94.9,112.0)\end{array}$ & $\begin{array}{l}100.0 \\
(91.0,100.6)\end{array}$ & $<.0001$ \\
\hline $\mathrm{FEV}_{1} \%$ predicted, $\%$ & $\begin{array}{l}97.5 \\
(88.2,105.8)\end{array}$ & $\begin{array}{l}91.9 \\
(82.1,101.1)\end{array}$ & $<.0001$ & $\begin{array}{l}101.0 \\
(93.4,109.2)\end{array}$ & $\begin{array}{l}97.0 \\
(88.2,106.0)\end{array}$ & $<.0001$ \\
\hline $\mathrm{FEV}_{1} / \mathrm{FVC}$ ratio & $\begin{array}{l}0.80 \\
(0.75,0.84)\end{array}$ & $\begin{array}{l}0.79 \\
(0.72,0.85)\end{array}$ & .009 & $\begin{array}{l}0.83 \\
(0.79,0.87)\end{array}$ & $\begin{array}{l}0.81 \\
(0.77,0.86)\end{array}$ & $<.0001$ \\
\hline PEF \% predicted, \% & $\begin{array}{l}95.5 \\
(85.6,105.5)\end{array}$ & $\begin{array}{l}92.0 \\
(81.4,103.6)\end{array}$ & $<.0001$ & $\begin{array}{l}103.8 \\
(93.0,114.7)\end{array}$ & $\begin{array}{l}103.6 \\
(92.5,115.2)\end{array}$ & .824 \\
\hline $\mathrm{FEF}_{50 \%} \%$ predicted, $\%$ & $\begin{array}{l}74.6 \\
(60.2,88.7)\end{array}$ & $\begin{array}{l}63.1 \\
(49.0,80.0)\end{array}$ & $<.0001$ & $\begin{array}{l}86.3 \\
(71.8,103.3)\end{array}$ & $\begin{array}{l}79.0 \\
(63.2,95.6)\end{array}$ & $<.0001$ \\
\hline $\mathrm{FEF}_{75 \%} \%$ predicted, $\%$ & $\begin{array}{l}55.8 \\
(40.7,71.0)\end{array}$ & $\begin{array}{l}49.5 \\
(35.6,67.6)\end{array}$ & $<.0001$ & $\begin{array}{l}65.0 \\
(50.7,84.4)\end{array}$ & $\begin{array}{l}62.0 \\
(46.4,82.1)\end{array}$ & $<.0001$ \\
\hline MMEF \% predicted, \% & $\begin{array}{l}65.8 \\
(52.6,81.4)\end{array}$ & $\begin{array}{l}59.2 \\
(44.2,76.8)\end{array}$ & $<.0001$ & $\begin{array}{l}77.5 \\
(64.1,92.4)\end{array}$ & $\begin{array}{l}72.7 \\
(57.5,88.7)\end{array}$ & $<.0001$ \\
\hline
\end{tabular}

Data are presented as absolute numbers in case of frequencies, median values, and quartiles in case of continuous parameters. Statistical comparisons based on percent predicted (pp) unless otherwise noted for pulmonary function test (PFT) values. $P$ values $<.05$ were considered to represent a significant difference in prevalence between SP signs of the negative and positive BPT categories

$\mathrm{BMI}$, body mass index; $\mathrm{FEV}_{1}$, forced expiratory volume in $1 \mathrm{~s} ; \mathrm{FEF}_{\mathrm{x} \%}$, instantaneous forced expiratory flow when $\mathrm{x} \%$ of the FVC has been expired; $\mathrm{MMEF}$, maximal midexpiratory flow

and FVC measurements $[1,20]$. Post-BPT and post$\mathrm{BDT}$ acceptable $\mathrm{FEV}_{1}$ and $\mathrm{FVC}$ measurements were also needed. A positive-BPT required a $20 \%$ fall from the post-diluent baseline $\mathrm{FEV}_{1}$ after the last dose of methacholine [21]. A positive-BDT was identified when the percent change from baseline and absolute changes in $\mathrm{FEV}_{1}$ and/or $\mathrm{FVC}$ values $\geq 12 \%$ and $\geq 200 \mathrm{ml}$ [2].

\section{Study design}

Four junior pulmonologists determined the SP sign based on a repeatable small plateau on the flow-volume curves. They independently labelled different portions of total curves (never the same) and all have experience in operating and interpreting spirometry for more than 2 years. If they have any doubts, then a senior pulmonologist with experience of more than 18 years would make the final decision. Representative examples of the SP sign were shown in Fig. 1, each BPT or BDT report has at least three flow-volume curves. Point $\mathrm{A}$ is the start point of the sign, point $B$ is the endpoint. The data points of A and B were extracted using Engauge Digitizer 11.1 software (M. Mitchell, Engauge Digitizer).
Volume (Vol) A/FVC and Flow A/PEF were original $(x, y)$ data of point $\mathrm{A}$; Vol $\mathrm{B} / \mathrm{FVC}$ and Flow $\mathrm{B} / \mathrm{PEF}$ were original ( $\mathrm{x}, \mathrm{y})$ data of point $\mathrm{B}$. ( Vol A - Vol B) /FVC and (Flow A - Flow B) /PEF represent the width and height of the sign, respectively. Pre-PFT questionnaires and electronic health records were reviewed by the same authors to identify symptoms and PFT indications. To further evaluate the features of the sign, according to the width or height of the sign, subjects were stratified into four classes, respectively:

Class 1: (Vol A - Vol B) $\times 100 / F V C$ ratio or (Flow A - Flow B) $\times 100 /$ PEF ratio $\leq 10 \%$

Class 2: $($ Vol A - Vol B) $\times 100 / F V C$ ratio or (Flow $\mathrm{A}-$ Flow B) $\times 100 /$ PEF ratio $>10 \%$ to $\leq 20 \%$

Class 3: (Vol A - Vol B) $\times 100 / F V C$ ratio or (Flow $\mathrm{A}-$ Flow B) $\times 100 /$ PEF ratio $>20 \%$ to $\leq 30 \%$

Class 4: (Vol A - Vol B) $\times 100 / F V C$ ratio or (Flow A - Flow B) $\times 100 /$ PEF ratio $>30 \%$

The study randomly selected 50 subjects with the sign from each class to compare the PFT and clinical features. 
Table 3 PFT values of patients with pre-BDTs

\begin{tabular}{|c|c|c|c|c|c|c|}
\hline \multirow[t]{2}{*}{ Parameter } & \multicolumn{3}{|l|}{ BDT $(+)$} & \multicolumn{3}{|l|}{ BDT $(-)$} \\
\hline & $\begin{array}{l}\text { SP sign }(+) \\
(N=25)\end{array}$ & $\begin{array}{l}S P \text { sign }(-) \\
(N=2904)\end{array}$ & $P$ value & $\begin{array}{l}S P \text { sign }(+) \\
(N=194)\end{array}$ & $\begin{array}{l}S P \operatorname{sign}(-) \\
(N=5044)\end{array}$ & $P$ value \\
\hline Age, years & $35(29,57)$ & $57.5(45,66)$ & $<.0001$ & $55(45,63)$ & $62(52,69)$ & $<.0001$ \\
\hline Sex, n (\%) & & & .014 & & & $<.0001$ \\
\hline Male & $12(48 \%)$ & 2047 (70\%) & & $105(54 \%)$ & $3554(70 \%)$ & \\
\hline Female & $13(52 \%)$ & 857 (30\%) & & $89(46 \%)$ & 1490 (30\%) & \\
\hline $\mathrm{BMI}, \mathrm{kg} / \mathrm{m}^{2}$ & $\begin{array}{l}22.5 \\
(19.9,24.2)\end{array}$ & $\begin{array}{l}22.5 \\
(19.8,25)\end{array}$ & .795 & $\begin{array}{l}23.7 \\
(21.4,26.0)\end{array}$ & $\begin{array}{l}22.2 \\
(19.5,24.8)\end{array}$ & $<.0001$ \\
\hline $\begin{array}{l}\text { Obesity, } \mathrm{n}(\%) \\
\left(\mathrm{BMI} \geq 30 \mathrm{~kg} / \mathrm{m}^{2}\right)\end{array}$ & 0/25 (0\%) & $96(3 \%)$ & 1.000 & $7 / 194$ (4\%) & 127 (3\%) & .347 \\
\hline FVC $\%$ predicted, $\%$ & $\begin{array}{l}92.8 \\
(82.9,100.5)\end{array}$ & $\begin{array}{l}78.1 \\
(65.1,91.0)\end{array}$ & $<.0001$ & $\begin{array}{l}101.1 \\
(88.5,112.5)\end{array}$ & $\begin{array}{l}80.0 \\
(66.8,94.4)\end{array}$ & $<.0001$ \\
\hline $\mathrm{FEV}_{1} \%$ predicted, $\%$ & $\begin{array}{l}72.1 \\
(63.9,78.5)\end{array}$ & $\begin{array}{l}51.0 \\
(35.9,64.6)\end{array}$ & $<.0001$ & $\begin{array}{l}88.4 \\
(78.5,97.0)\end{array}$ & $\begin{array}{l}58.0 \\
(40.0,74.0)\end{array}$ & $<.0001$ \\
\hline $\mathrm{FEV}_{1} / \mathrm{FVC}$ ratio & $\begin{array}{l}0.67 \\
(0.62,0.71)\end{array}$ & $\begin{array}{l}0.52 \\
(0.42,0.63)\end{array}$ & $<.0001$ & $\begin{array}{l}0.73 \\
(0.68,0.77)\end{array}$ & $\begin{array}{l}0.59 \\
(0.44,0.69)\end{array}$ & $<.0001$ \\
\hline PEF \% predicted, \% & $\begin{array}{l}76.1 \\
(69.5,84.4)\end{array}$ & $\begin{array}{l}52.6 \\
(36.8,69.4)\end{array}$ & $<.0001$ & $\begin{array}{l}90.9 \\
(78.5,102.7)\end{array}$ & $\begin{array}{l}62.1 \\
(42.0,82.5)\end{array}$ & $<.0001$ \\
\hline $\mathrm{FEF}_{50 \%} \%$ predicted, $\%$ & $\begin{array}{l}38.5 \\
(27.0,47.3)\end{array}$ & $\begin{array}{l}18.0 \\
(10.3,30.2)\end{array}$ & $<.0001$ & $\begin{array}{l}52.9 \\
(42.9,67.1)\end{array}$ & $\begin{array}{l}23.8 \\
(11.9,40.8)\end{array}$ & $<.0001$ \\
\hline $\mathrm{FEF}_{75 \%} \%$ predicted, $\%$ & $\begin{array}{l}24.2 \\
(18.5,33.5)\end{array}$ & $\begin{array}{l}15.5 \\
(10.8,23.0)\end{array}$ & $<.0001$ & $\begin{array}{l}31.3 \\
(23.1,43.0)\end{array}$ & $\begin{array}{l}19.4 \\
(12.6,29.7)\end{array}$ & $<.0001$ \\
\hline MMEF \% predicted, $\%$ & $\begin{array}{l}30.9 \\
(21.1,40.6)\end{array}$ & $\begin{array}{l}15.8 \\
(9.7,25.2)\end{array}$ & $<.0001$ & $\begin{array}{l}44.2 \\
(34.4,53.6)\end{array}$ & $\begin{array}{l}20.5 \\
(11.3,34.9)\end{array}$ & $<.0001$ \\
\hline
\end{tabular}

Data are presented as absolute numbers in case of frequencies, median values, and quartiles in case of continuous parameters. Statistical comparisons based on percent predicted (pp) unless otherwise noted for pulmonary function test (PFT) values. $P$ values $<.05$ were considered to represent a significant difference in prevalence between SP signs of the negative and positive BDT categories

PFT, pulmonary function test; BMI, body mass index; BDT, bronchodilator test; SP, small plateau; FVC, forced vital capacity; PEF, peak expiratory flow; FEV 1 , forced expiratory volume in $1 \mathrm{~s} ; \mathrm{FEF}_{\mathrm{x} \%}$, instantaneous forced expiratory flow when $\mathrm{x} \%$ of the FVC has been expired; MMEF, maximal mid-expiratory flow

All the electronic health records were reviewed within 6 months of PFTs.

For patients who underwent laryngoscopy according to specialists' decision in the department of otolaryngology, FS tests were performed after laryngoscopy was completed. Patients who had contraindications for PFTs were excluded according to the Chinese Thoracic Society guidelines [22].

\section{Development of the model}

3,453 (1953 SP sign positive and 1500 negative samples) and 374 samples (95 positive and 279 negative samples) were used for training and testing the model, respectively. 294 positive samples could not be used due to the model failure to extract the image. Inputs to the model were images extracted from collected PFT reports in pdf. format, to be specific, flow-volume curves were saved as vector graphics and could be extracted by developing Python scripts. All extracted curves were further processed to have a pixel size of $600 * 1200$ by using the OpenCV-Python package. A couple of examples of pdf and curve images can be found in Fig. 2. Each PFT report must have at least three flow-volume curves, only when repeatable (at least three curves) detecting of the SP sign, then the model would output the presence of the sign. In this work, the recognition of the SP sign was formulated as a detection task. To this end, we proposed SP Network (SP-Net), a Faster R-CNN [18] alike object detection model.

As shown in Fig. 3, given an input image, the classic ResNet50 [17] convolutional neural network was utilized to extract the feature map. Then a region proposal network would generate object bounds and objectness scores, i.e., the probability of a specific sign occurs and its position, according to the feature map in a sliding window fashion. Next, a region of interest pooling layer would extract a feature vector from the feature map for each of the proposals. Each feature vector was fed into a series of fully connected layers that finally branch into a classifier and a regressor which output: (1) Whether an SP sign was detected; (2) Bounding box positions.

The model was firstly trained for 20,000 iterations with a learning rate of $1 \mathrm{e}-3$ and then trained for another 
Table 4 Pre-PFT questionnaires of BPTS

\begin{tabular}{|c|c|c|c|c|c|c|}
\hline \multirow[t]{2}{*}{ Parameter } & \multicolumn{2}{|l|}{ BPT (+) } & \multirow[t]{2}{*}{$P$ value } & \multicolumn{2}{|l|}{ BPT (-) } & \multirow[t]{2}{*}{$P$ value } \\
\hline & $\begin{array}{l}\mathrm{SP} \text { sign }(+) \\
(\mathrm{N}=205)\end{array}$ & $\begin{array}{l}\text { SP sign }(-) \\
(\mathrm{N}=3961)\end{array}$ & & $\begin{array}{l}\text { SP sign }(+) \\
(\mathrm{N}=1124)\end{array}$ & $\begin{array}{l}\text { SP sign (-) } \\
(\mathrm{N}=4624)\end{array}$ & \\
\hline Smoking status, n (\%) & & & .348 & & & $<.0001$ \\
\hline Current or former smoker & $23(11 \%)$ & $535(14 \%)$ & & 155 (15\%) & 888 (19\%) & \\
\hline Never smoker & $182(89 \%)$ & $3426(86 \%)$ & & $969(86 \%)$ & $3753(81 \%)$ & \\
\hline Chronic cough, n (\%) & & & $<.0001$ & & & $<.0001$ \\
\hline Rare & $49(24 \%)$ & $1428(36 \%)$ & & $171(15 \%)$ & $1176(25 \%)$ & \\
\hline Sometimes & $156(76 \%)$ & $2533(64 \%)$ & & $947(85 \%)$ & $3448(75 \%)$ & \\
\hline Sputum production, n (\%) & $\begin{array}{l}105 / 205 \\
(51 \%)\end{array}$ & $\begin{array}{l}\text { 1967/3961 } \\
(50 \%)\end{array}$ & .663 & $\begin{array}{l}568 / 1124 \\
(51 \%)\end{array}$ & $\begin{array}{l}2478 / 4624 \\
(54 \%)\end{array}$ & .066 \\
\hline Wheezing, n (\%) & $\begin{array}{l}77 / 205 \\
(38 \%)\end{array}$ & $\begin{array}{l}1209 / 3961 \\
(31 \%)\end{array}$ & .033 & $\begin{array}{l}342 / 1124 \\
(30 \%)\end{array}$ & $\begin{array}{l}865 / 4624 \\
(19 \%)\end{array}$ & $<.0001$ \\
\hline \multicolumn{7}{|l|}{ PFT indication, n (\%) } \\
\hline Cough & $\begin{array}{l}61 / 205 \\
(30 \%)\end{array}$ & $\begin{array}{l}\text { 1345/3961 } \\
(34 \%)\end{array}$ & .360 & $\begin{array}{l}663 / 1124 \\
(59 \%)\end{array}$ & $\begin{array}{l}2494 / 4624 \\
(54 \%)\end{array}$ & $<.0001$ \\
\hline COPD & $\begin{array}{l}2 / 205 \\
(1 \%)\end{array}$ & $\begin{array}{l}152 / 3961 \\
(4 \%)\end{array}$ & .034 & $\begin{array}{l}64 / 1124 \\
(6 \%)\end{array}$ & $\begin{array}{l}237 / 4624 \\
(5 \%)\end{array}$ & .443 \\
\hline Asthma & $\begin{array}{l}162 / 205 \\
(79 \%)\end{array}$ & $\begin{array}{l}2098 / 3961 \\
(53 \%)\end{array}$ & $<.0001$ & $\begin{array}{l}162 / 1124 \\
(14 \%)\end{array}$ & $\begin{array}{l}675 / 4624 \\
(15 \%)\end{array}$ & .875 \\
\hline Bronchiectasis & $\begin{array}{l}5 / 205 \\
(2 \%)\end{array}$ & $\begin{array}{l}26 / 3961 \\
(1 \%)\end{array}$ & .016 & $\begin{array}{l}46 / 1124 \\
(14 \%)\end{array}$ & $\begin{array}{l}90 / 4624 \\
(2 \%)\end{array}$ & $<.0001$ \\
\hline ILD & $\begin{array}{l}0 / 205 \\
(0 \%)\end{array}$ & $\begin{array}{l}17 / 3961 \\
(0 \%)\end{array}$ & 1.000 & $\begin{array}{l}10 / 1124 \\
(1 \%)\end{array}$ & $\begin{array}{l}55 / 4624 \\
(1 \%)\end{array}$ & .394 \\
\hline Rhinitis/sinusitis & $\begin{array}{l}50 / 205 \\
(24 \%)\end{array}$ & $\begin{array}{l}46 / 3961 \\
(1 \%)\end{array}$ & $<.0001$ & $\begin{array}{l}147 / 1124 \\
(13 \%)\end{array}$ & $\begin{array}{l}50 / 4624 \\
(1 \%)\end{array}$ & $<.0001$ \\
\hline Gastroesophageal reflux & $\begin{array}{l}2 / 205 \\
(1 \%)\end{array}$ & $\begin{array}{l}1 / 3961 \\
(0.03 \%)\end{array}$ & .007 & $\begin{array}{l}15 / 1124 \\
(1 \%)\end{array}$ & $\begin{array}{l}3 / 4624 \\
(0 \%)\end{array}$ & $<.0001$ \\
\hline Pneumonia & $\begin{array}{l}10 / 205 \\
(5 \%)\end{array}$ & $\begin{array}{l}29 / 3961 \\
(0.7 \%)\end{array}$ & $<.0001$ & $\begin{array}{l}58 / 1124 \\
(5 \%)\end{array}$ & $\begin{array}{l}102 / 4624 \\
(2 \%)\end{array}$ & $<.0001$ \\
\hline Shortness of breath & $\begin{array}{l}5 / 205 \\
(2 \%)\end{array}$ & $\begin{array}{l}65 / 3961 \\
(2 \%)\end{array}$ & .393 & $\begin{array}{l}43 / 1124 \\
(4 \%)\end{array}$ & $\begin{array}{l}143 / 4624 \\
(3 \%)\end{array}$ & .213 \\
\hline Chest distress & $\begin{array}{l}4 / 205 \\
(2 \%)\end{array}$ & $\begin{array}{l}35 / 3961 \\
(1 \%)\end{array}$ & .123 & $\begin{array}{l}29 / 1124 \\
(3 \%)\end{array}$ & $\begin{array}{l}121 / 4624 \\
(3 \%)\end{array}$ & .945 \\
\hline Others $^{\mathrm{a}}$ & $\begin{array}{l}4 / 205 \\
(2 \%)\end{array}$ & $\begin{array}{l}147 / 3961 \\
(4 \%)\end{array}$ & 1.000 & $\begin{array}{l}7 / 1124 \\
(1 \%)\end{array}$ & $\begin{array}{l}654 / 4624 \\
(14 \%)\end{array}$ & $<.0001$ \\
\hline
\end{tabular}

Data are presented as absolute numbers in the case of frequencies. $P$ values $<.05$ were considered to represent a significant difference in prevalence between SP signs of the negative and positive BPT

PFT, pulmonary function test; COPD, chronic obstructive pulmonary disease; ILD, interstitial lung disease

${ }^{a}$ Indications include hypertension, coronary heart disease, diabetes, eosinophilic granulomatosis with polyangiitis, and studies without documented indication

10,000 iterations with a smaller learning rate of $1 \mathrm{e}-4$. The batch size was set to 1 through all iterations. A stochastic gradient descent optimizer was utilized to train the model. Our training objective function was a multi-task loss following Fast R-CNN [23], which consisted of a classification loss and a regression loss for calculating the difference between model predictions and ground truth values.

\section{Statistical analysis}

Count data were analyzed by using the $\chi^{2}$ or the Fischer exact test, as appropriate. Median comparisons were performed by using the Kruskal-Wallis test, or MannWhitney U test when there were two groups. Mean comparisons were performed by using the One-Way ANOVA or independent-samples T-test, as appropriate.

The deep learning model was implemented using TensorFlow 1.14.0 and trained on 4 Tesla K80 GPUs. Other statistical analyses were performed with SPSS version 26.

\section{Results}

Of the 13,661 BPTs reviewed, 2,123 (15.5\%) patients with SP sign were labelled. Of these, 1,782 (83.9\%) were negative-BPT; 341 (16.1\%) were positive-BPT (Fig. 4). Of a 
Table 5 Pre-PFT questionnaires of BDTs

\begin{tabular}{|c|c|c|c|c|c|c|}
\hline \multirow[t]{2}{*}{ Parameter } & \multicolumn{3}{|l|}{ BDT (+) } & \multicolumn{3}{|l|}{ BDT (-) } \\
\hline & $\begin{array}{l}S P \text { sign }(+) \\
(\mathrm{N}=12)\end{array}$ & $\begin{array}{l}\text { SP sign (-) } \\
(\mathrm{N}=2235)\end{array}$ & $P$ value & $\begin{array}{l}S P \text { sign }(+) \\
(\mathrm{N}=119)\end{array}$ & $\begin{array}{l}\text { SP sign (-) } \\
(\mathrm{N}=3728)\end{array}$ & $P$ value \\
\hline Smoking status, n (\%) & & & .111 & & & .005 \\
\hline Current or former smoker & $3(25 \%)$ & $1083(48 \%)$ & & $45(38 \%)$ & 1929 (52\%) & \\
\hline Never smoker & $9(75 \%)$ & $1172(52 \%)$ & & $74(62 \%)$ & $1799(48 \%)$ & \\
\hline Chronic cough, n (\%) & & & .338 & & & .270 \\
\hline Rare & $2(17 \%)$ & $625(28 \%)$ & & 27 (23\%) & $1016(27 \%)$ & \\
\hline Sometimes & $10(83 \%)$ & $1610(72 \%)$ & & $92(77 \%)$ & $2712(73 \%)$ & \\
\hline Sputum production, n (\%) & $\begin{array}{l}9 / 12 \\
(75 \%)\end{array}$ & $\begin{array}{l}1559 / 2235 \\
(70 \%)\end{array}$ & 1.000 & $\begin{array}{l}75 / 119 \\
(63 \%)\end{array}$ & $\begin{array}{l}2651 / 3728 \\
(71 \%)\end{array}$ & .056 \\
\hline Wheezing, n (\%) & $\begin{array}{l}11 / 12 \\
(92 \%)\end{array}$ & $\begin{array}{l}1234 / 2235 \\
(55 \%)\end{array}$ & .011 & $\begin{array}{l}68 / 119 \\
(57 \%)\end{array}$ & $\begin{array}{l}1703 / 3728 \\
(46 \%)\end{array}$ & .014 \\
\hline \multicolumn{7}{|l|}{ PFT indication, n (\%) } \\
\hline Cough & $\begin{array}{l}2 / 12 \\
(17 \%)\end{array}$ & $\begin{array}{l}262 / 2235 \\
(12 \%)\end{array}$ & .643 & $\begin{array}{l}8 / 119 \\
(7 \%)\end{array}$ & $\begin{array}{l}328 / 3728 \\
(9 \%)\end{array}$ & .430 \\
\hline COPD & $\begin{array}{l}0 / 12 \\
(0 \%)\end{array}$ & $\begin{array}{l}809 / 2235 \\
(34 \%)\end{array}$ & .006 & $\begin{array}{l}33 / 119 \\
(28 \%)\end{array}$ & $\begin{array}{l}1696 / 3728 \\
(45 \%)\end{array}$ & $<.0001$ \\
\hline Asthma & $\begin{array}{l}10 / 12 \\
(83 \%)\end{array}$ & $\begin{array}{l}905 / 2235 \\
(40 \%)\end{array}$ & .002 & $\begin{array}{l}38 / 119 \\
(32 \%)\end{array}$ & $\begin{array}{l}720 / 3728 \\
(19 \%)\end{array}$ & $<.0001$ \\
\hline Bronchiectasis & $\begin{array}{l}0 / 12 \\
(0 \%)\end{array}$ & $\begin{array}{l}54 / 2235 \\
(2 \%)\end{array}$ & 1.000 & $\begin{array}{l}11 / 119 \\
(9 \%)\end{array}$ & $\begin{array}{l}293 / 3728 \\
(8 \%)\end{array}$ & .582 \\
\hline ILD & $\begin{array}{l}0 / 12 \\
(0 \%)\end{array}$ & $\begin{array}{l}12 / 2235 \\
(1 \%)\end{array}$ & 1.000 & $\begin{array}{l}3 / 119 \\
(3 \%)\end{array}$ & $\begin{array}{l}70 / 3728 \\
(2 \%)\end{array}$ & .493 \\
\hline Rhinitis/sinusitis & $\begin{array}{l}3 / 12 \\
(25 \%)\end{array}$ & $\begin{array}{l}18 / 2235 \\
(1 \%)\end{array}$ & $<.0001$ & $\begin{array}{l}21 / 119 \\
(18 \%)\end{array}$ & $\begin{array}{l}39 / 3728 \\
(1 \%)\end{array}$ & $<.0001$ \\
\hline Gastroesophageal reflux & $\begin{array}{l}0 / 12 \\
(0 \%)\end{array}$ & $\begin{array}{l}1 / 2235 \\
(0 \%)\end{array}$ & 1.000 & $\begin{array}{l}3 / 119 \\
(3 \%)\end{array}$ & $\begin{array}{l}0 / 3728 \\
(0 \%)\end{array}$ & $<.0001$ \\
\hline Pneumonia & $\begin{array}{l}3 / 12 \\
(25 \%)\end{array}$ & $\begin{array}{l}56 / 2235 \\
(3 \%)\end{array}$ & .003 & $\begin{array}{l}16 / 119 \\
(13 \%)\end{array}$ & $\begin{array}{l}210 / 3728 \\
(6 \%)\end{array}$ & $<.0001$ \\
\hline Shortness of breath & $\begin{array}{l}1 / 12 \\
(8 \%)\end{array}$ & $\begin{array}{l}114 / 2235 \\
(5 \%)\end{array}$ & .468 & $\begin{array}{l}5 / 119 \\
(4 \%)\end{array}$ & $\begin{array}{l}176 / 3728 \\
(5 \%)\end{array}$ & .792 \\
\hline Chest distress & $\begin{array}{l}0 / 12 \\
(0 \%)\end{array}$ & $\begin{array}{l}9 / 2235 \\
(0 \%)\end{array}$ & 1.000 & $\begin{array}{l}2 / 119 \\
(2 \%)\end{array}$ & $\begin{array}{l}25 / 3728 \\
(1 \%)\end{array}$ & .203 \\
\hline Others $^{\mathrm{a}}$ & $\begin{array}{l}0 / 12 \\
(0 \%)\end{array}$ & $\begin{array}{l}48 / 2235 \\
(2 \%)\end{array}$ & 1.000 & $\begin{array}{l}5 / 119 \\
(4 \%)\end{array}$ & $\begin{array}{l}171 / 3728 \\
(5 \%)\end{array}$ & .843 \\
\hline
\end{tabular}

Data are presented as absolute numbers in the case of frequencies. $P$ values $<.05$ were considered to represent a significant difference in prevalence between $\mathrm{SP}$ signs of the negative and positive BDT categories

COPD, chronic obstructive pulmonary disease; ILD, interstitial lung disease

${ }^{a}$ Indications include hypertension, coronary heart disease, diabetes, eosinophilic granulomatosis with polyangiitis, and studies without documented indication

total 8,168 BDTs reviewed, there were 219 (2.7\%) labeled as the SP sign. Of these, $194(88.6 \%)$ were negative-BDT, 25 (11.4\%) were positive- BDT (Fig. 5).

\section{Characteristic PFT parameters}

SP sign was calculated mostly located on the $90-70 \%$ PEF and $18-40 \%$ FVC expiratory phase. Compared with positive-BPT, subjects with a negative-BPT had a more obvious SP sign which appeared to be wider and higher in the pre-challenge spirometry (both $P<0.0001$ ) (Table 1 ). Most of the width of the sign of subjects with a positiveBPT narrowed or even disappeared in the post-BPT. On the contrary, subjects with a positive-BDT mostly without the sign in the prebronchodilator spirometry, the sign appeared in the post-bronchodilator maneuvers. (Table 1).

Of the negative-BPT group, patients with SP sign were more often female and had a prevalence of obesity $\left(\mathrm{BMI} \geq 30 \mathrm{~kg} / \mathrm{m}^{2}\right)$ (both, $\left.P<0.0001\right)$ (Table 2$)$. In pre-BPT and pre-BDT groups, compared patients without SP sign, patients with SP sign had higher median $\mathrm{FVC}, \mathrm{FEV}_{1}, \mathrm{FEF}_{50 \%}, \mathrm{FEF}_{75 \%}$, and $\mathrm{MMEF} \%$ predicted (all $P<0.0001$ ); patients with the sign also had a higher 
Table 6 Baseline characteristics of the SP width-stratified classes in negative pre-BPTs

\begin{tabular}{|c|c|c|c|c|c|}
\hline \multirow[t]{2}{*}{ Parameter } & \multicolumn{5}{|c|}{ SP sign of negative pre-BPTs } \\
\hline & $\begin{array}{l}(\text { Vol } A-\text { Vol B }) \times 100 / \\
\text { FVC ratio } \\
(\leq 10 \%) \\
(\mathrm{N}=50)\end{array}$ & $\begin{array}{l}(\text { Vol } A-\text { Vol B }) \times 100 / \\
\text { FVC ratio } \\
(>10 \% \text { to } \leq 20 \%) \\
(N=50)\end{array}$ & $\begin{array}{l}(\text { Vol } A-\text { Vol B }) \times 100 / \\
\text { FVC ratio } \\
(>20 \% \text { to } \leq 30 \%) \\
(N=50)\end{array}$ & $\begin{array}{l}(\mathrm{Vol} A-\mathrm{Vol} \mathrm{B}) \times 100 / \\
\text { FVC ratio } \\
(>30 \%) \\
(\mathrm{N}=50)\end{array}$ & $P$ value \\
\hline Age, years & $39.1 \pm 2.2$ & $41.9 \pm 2.2$ & $40.6 \pm 1.8$ & $37.7 \pm 2.0$ & .797 \\
\hline Sex, n (\%) & & & & & .002 \\
\hline Male & $31(62 \%)^{c, d}$ & $25(50 \%)$ & $14(28 \%)^{\mathrm{a}}$ & $17(34 \%)^{\mathrm{a}}$ & \\
\hline Female & $19(38 \%)^{c, d}$ & $25(50 \%)$ & $36(72 \%)^{\mathrm{a}}$ & $33(66 \%)^{\mathrm{a}}$ & \\
\hline $\mathrm{BMI}, \mathrm{kg} / \mathrm{m}^{2}$ & $22.4(19.8,24.3)$ & $22.4(19.9,24.5)$ & $22.4(20.2,25.2)$ & $21.3(20.2,24.6)$ & .884 \\
\hline $\begin{array}{l}\text { Obesity, } n(\%) \\
\left(\mathrm{BMl} \geq 30 \mathrm{~kg} / \mathrm{m}^{2}\right)\end{array}$ & 0/50 (0\%) & $2 / 50(4 \%)$ & $2 / 50(4 \%)$ & $2 / 50(4 \%)$ & .727 \\
\hline $\begin{array}{l}\text { Underweight, n (\%) } \\
\left(\mathrm{BMl}<18.5 \mathrm{~kg} / \mathrm{m}^{2}\right)\end{array}$ & $5 / 50(10 \%)$ & $5 / 50(10 \%)$ & $5 / 50(10 \%)$ & $5 / 50(10 \%)$ & 1.000 \\
\hline Smoking status, n (\%) & & & & & .005 \\
\hline Current or former smoker & $17(34 \%)^{d}$ & $7(14 \%)$ & $5(10 \%)$ & $6(12 \%)^{\mathrm{a}}$ & \\
\hline Never smoker & $33(66 \%)^{d}$ & $43(86 \%)$ & 45 (90\%) & $44(88 \%)^{\mathrm{a}}$ & \\
\hline FVC $\%$ predicted, $\%$ & $102.5(95.3,108.2)$ & $101.7(92.4,111.1)$ & $104.1(98.1,113.3)$ & $106.3(95.1,115.4)$ & .200 \\
\hline $\mathrm{FEV}_{1} \%$ predicted, $\%$ & $97.7(91.9,103.3)^{d}$ & $98.0(91.9,108.0)$ & $102.6(96.0,112.5)$ & $105.2(98.1,111.4)^{\mathrm{a}}$ & .007 \\
\hline $\mathrm{FEV}_{1} / \mathrm{FVC}$ ratio & $0.81 \pm 0.01^{d}$ & $0.82 \pm 0.01$ & $0.83 \pm 0.01$ & $0.85 \pm 0.01^{\mathrm{a}}$ & .003 \\
\hline Chronic cough, n (\%) & & & & & $<.0001$ \\
\hline Rare & $6(12 \%)^{d}$ & $11(22 \%)$ & $5(10 \%)$ & $26(52 \%)^{\mathrm{a}}$ & \\
\hline Sometimes & $44(88 \%)^{d}$ & $39(78 \%)$ & 45 (90\%) & $24(48 \%)^{\mathrm{a}}$ & \\
\hline Sputum production, n (\%) & $24 / 50(48 \%)$ & $24 / 50(48 \%)$ & $22 / 50(44 \%)$ & $24 / 50(48 \%)$ & .971 \\
\hline Chest tightness, n (\%) & 0/50 (0\%) & 0/50 (0\%) & $0 / 50(0 \%)$ & $1 / 50(2 \%)$ & 1.000 \\
\hline Wheezing, n (\%) & $15 / 50(30 \%)$ & $14 / 50(28 \%)$ & $8 / 50(16 \%)$ & $16 / 50(32 \%)$ & .264 \\
\hline \multicolumn{6}{|l|}{ PFT indication, $\mathrm{n}(\%)$} \\
\hline Asthma & $7(14 \%)$ & $8(16 \%)$ & $6(12 \%)$ & $2(4 \%)$ & .253 \\
\hline COPD & $10(20 \%)^{d}$ & $3(6 \%)$ & $0(0 \%)$ & $1(2 \%)^{\mathrm{a}}$ & $<.0001$ \\
\hline ILD & $0(0 \%)$ & $2(4 \%)$ & $0(0 \%)$ & $0(0 \%)$ & .246 \\
\hline Pulmonary nodule/neoplasia & $1(2 \%)$ & $2(4 \%)$ & $1(2 \%)$ & $0(0 \%)$ & .903 \\
\hline Cough & $26(52 \%)$ & $28(56 \%)$ & $28(56 \%)$ & $29(58 \%)$ & .943 \\
\hline Shortness of breath & $1(2 \%)$ & $1(2 \%)$ & $2(4 \%)$ & $3(6 \%)$ & .839 \\
\hline Rhinitis/sinusitis & $5(10 \%)$ & $2(4 \%)$ & $5(10 \%)$ & $2(4 \%)$ & .477 \\
\hline Other $^{\mathrm{e}}$ & $0(0 \%)^{d}$ & $4(8 \%)$ & $8(16 \%)$ & $13(26 \%)^{\mathrm{a}}$ & .001 \\
\hline
\end{tabular}

Data are presented as absolute numbers (percentages) in case of frequencies, median values, and quartiles in case of continuous parameters unless otherwise noted. Statistical comparisons based on percent predicted (pp) unless otherwise noted for pulmonary function test (PFT) values

a $P<0.05 ;($ Vol $A-$ Vol B $) \times 100 / F V C$ ratio $\leq 10 \%$ versus the remaining groups

${ }^{b} P<0.05 ;(\mathrm{Vol} A-\mathrm{Vol} B) \times 100 / F V C$ ratio $>10 \%$ to $\leq 20 \%$ versus the remaining groups

${ }^{c} P<0.05 ;(\mathrm{Vol} A-\mathrm{Vol} B) \times 100 / \mathrm{FVC}$ ratio $>20 \%$ to $\leq 30 \%$ versus the remaining groups

${ }^{d} P<0.05 ;($ Vol A - Vol B) $\times 100 / F V C$ ratio $>30 \%$ versus the remaining groups

e Indications include bronchiectasis, chest pain, pleural effusion, sleep disorder, and studies without documented indication

median $\mathrm{FEV}_{1} / \mathrm{FVC}$ ratio $(P=0.009, P<0.0001, P<0.0001$ and $P<0.0001$, respectively) (Tables 2 and 3 ).

\section{Pre-PFT questionnaires}

Patients with SP sign had higher rates of chronic cough in BPT groups (both $P<0.0001$ ) (Table 4). In addition, patients with the sign were more likely to have evidence of wheezing in positive and/or negative BPT and BDT groups $(P=0.033, P<0.0001, P=0.011$ and $P=0.014$, respectively) (Tables 4 and 5).

In negative-BPT groups, patients with the sign were more likely to have PFT indications of cough, 
Table 7 Baseline characteristics of the SP height-stratified classes in negative pre-BPTs

\begin{tabular}{|c|c|c|c|c|c|}
\hline \multirow[t]{2}{*}{ Parameter } & \multicolumn{5}{|c|}{ SP sign of negative pre-BPTs } \\
\hline & $\begin{array}{l}(\text { Flow } A-\text { Flow } B) \times 100 / \\
\text { PEF ratio } \\
(\leq 10 \%) \\
(\mathrm{N}=50)\end{array}$ & $\begin{array}{l}(\text { Flow } A-\text { Flow } B) \times 100 / \\
\text { PEF ratio } \\
(>10 \% \text { to } \leq 20 \%) \\
(N=50)\end{array}$ & $\begin{array}{l}(\text { Flow } A-\text { Flow } B) \times 100 / \\
\text { PEF ratio } \\
(>20 \% \text { to } \leq 30 \%) \\
(N=50)\end{array}$ & $\begin{array}{l}(\text { Flow } A-\text { Flow } B) \times 100 / \\
\text { PEF ratio } \\
(>30 \%) \\
(N=50)\end{array}$ & $P$ value \\
\hline Age, years & $39.1 \pm 2.2$ & $41.9 \pm 2.2$ & $40.6 \pm 1.8$ & $37.7 \pm 2.0$ & .119 \\
\hline Sex, n (\%) & & & & & .262 \\
\hline Male & $29(58 \%)$ & $16(32 \%)$ & $26(52 \%)$ & $13(26 \%)$ & \\
\hline Female & $21(42 \%)$ & $34(68 \%)$ & $24(48 \%)$ & $37(74 \%)$ & \\
\hline $\mathrm{BMI}, \mathrm{kg} / \mathrm{m}^{2}$ & $23.5(21.1,26.3)$ & $23.1(20.5,25.3)$ & $22.3(19.8,24.9)$ & $21.3(20.2,24.6)$ & .156 \\
\hline FVC $\%$ predicted, $\%$ & $104.9 \pm 2.0$ & $103.1 \pm 1.8$ & $103.6 \pm 1.80$ & $107.1 \pm 1.8$ & .408 \\
\hline $\mathrm{FEV}, \%$ predicted, $\%$ & $100.9 \pm 1.6$ & $99.1 \pm 1.6^{d}$ & $101.6 \pm 1.4$ & $106.4 \pm 1.7^{b}$ & .009 \\
\hline $\mathrm{FEV}_{1} / \mathrm{FVC}$ ratio & $1.01(0.94,1.09)^{b, c, d}$ & $0.81(0.79,0.84)^{\mathrm{a}}$ & $0.84(0.80,0.87)^{\mathrm{a}}$ & $0.85(0.81,0.88)^{\mathrm{a}}$ & .000 \\
\hline
\end{tabular}

Data are presented as absolute numbers (percentages) in case of frequencies, median values, and quartiles in case of continuous parameters unless otherwise noted. Statistical comparisons based on percent predicted ( $\mathrm{pp}$ ) unless otherwise noted for pulmonary function test (PFT) values

a $P<0.05$; (Flow $A-$ Flow $B) \times 100 / P E F$ ratio $\leq 10 \%$ versus the remaining groups

${ }^{\mathrm{b}} P<0.05$; (Flow $A-$ Flow $B$ ) $\times 100 / P E F$ ratio $>10 \%$ to $\leq 20 \%$ versus the remaining groups

${ }^{c} P<0.05$; (Flow $A-$ Flow $B$ ) $\times 100 / P E F$ ratio $>20 \%$ to $\leq 30 \%$ versus the remaining groups

${ }^{d} P<0.05$; (Flow $A-$ Flow $B$ ) $\times 100 /$ PEF ratio $>30 \%$ versus the remaining groups

Table 8 Laryngoscopy findings associated with the SP sign

\begin{tabular}{|c|c|c|c|}
\hline Parameter & $\begin{array}{l}\text { SP sign }(+) \\
(n=16)\end{array}$ & $\begin{array}{l}\text { SP sign }(-) \\
(n=32)\end{array}$ & $P$ value \\
\hline Age, years & $44.2 \pm 3.1$ & $50.0 \pm 3.0$ & .223 \\
\hline Sex, n (\%) & & & .147 \\
\hline Male & $7(44 \%)$ & $21(66 \%)$ & \\
\hline Female & $9(56 \%)$ & $11(34 \%)$ & \\
\hline $\mathrm{BMI}, \mathrm{kg} / \mathrm{m}^{2}$ & $25.0 \pm 1.0$ & $22.5 \pm 0.8$ & .060 \\
\hline Smoking status, n (\%) & & & .404 \\
\hline Current or former smoker & $5(31 \%)$ & $14(44 \%)$ & \\
\hline Never smoker & $11(69 \%)$ & $18(56 \%)$ & \\
\hline \multicolumn{4}{|l|}{ Laryngoscopy finding, n (\%) } \\
\hline Epiglottis cyst & $2(13 \%)$ & $0(0 \%)$ & .106 \\
\hline Vocal cord/throat nodule/tumor & $4(25 \%)$ & $7(22 \%)$ & 1.000 \\
\hline $\begin{array}{l}\text { Hypertrophy of tonsils/ } \\
\text { adenoids }\end{array}$ & $4(25 \%)$ & $3(9 \%)$ & .195 \\
\hline Chronic pharyngitis/normal & $6(37 \%)$ & 22 (69\%) & .038 \\
\hline
\end{tabular}

Data are presented as absolute numbers (percentages) in case of frequencies unless otherwise noted bronchiectasis, rhinitis (sinusitis), gastroesophageal reflux and pneumonia (all $P<0.0001$ ) (Table 4). In positive-BDT groups, PFT indications of asthma, rhinitis (sinusitis), and pneumonia had higher rates, but COPD had a lower rate within the sign $(P=0.002, P<0.0001$, $P=0.003$, and $P=0.006$, respectively); patients with the sign had higher rates of PFT indications of asthma, rhinitis (sinusitis), gastroesophageal reflux and pneumonia in the negative-BDT group (all $P<0.0001$ ), on the contrary, a lower rate of PFT indication of COPD $(P<0.0001)$ (Table 5).

\section{Baseline characteristics of the SP sign classes}

In the width-stratified classes, of 1,782 subjects, there were $245(13.7 \%)$ with class $1,652(36.6 \%)$ with class 2 , $507(28.5 \%)$ with class 3, and $378(21.2 \%)$ subjects with class 4 (Fig. 4). Compared with class 1 , subjects with class 4 were more often never smoker $(P=0.005)$ and with higher median $\mathrm{FEV}_{1} \%$ predicted and $\mathrm{FEV}_{1} / \mathrm{FVC}$ ratio
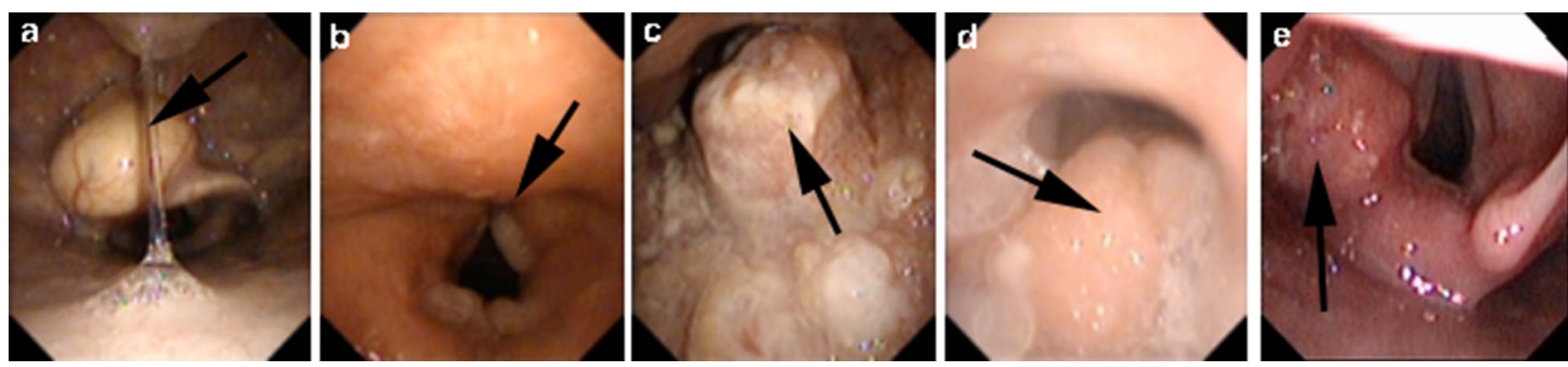

Fig. 6 Examples of the laryngoscopy findings. a Epiglottic cyst; $\mathbf{b}$ vocal cord nodule; c tonsil mass, $\mathbf{d}$ hypertrophy of tonsils/adenoids; e supraglottic laryngeal cancer. The location indicated by the black arrow is the lesion 
Table 9 Precision data summary

\begin{tabular}{lll}
\hline Degree of stenosis & $\begin{array}{l}\text { SP sign }(+) \\
(\mathbf{N}=10)\end{array}$ & $\begin{array}{l}\text { SP sign }(-) \\
(\mathbf{N}=10)\end{array}$ \\
\hline$\sim 25 \%$ & 10 & 5 \\
$50 \%$ & 0 & 2 \\
$75 \%$ & 0 & 1 \\
$90 \%$ & 0 & 2 \\
\hline
\end{tabular}

Data are presented as absolute numbers in the case of frequencies $\mathrm{SP}$, small plateau

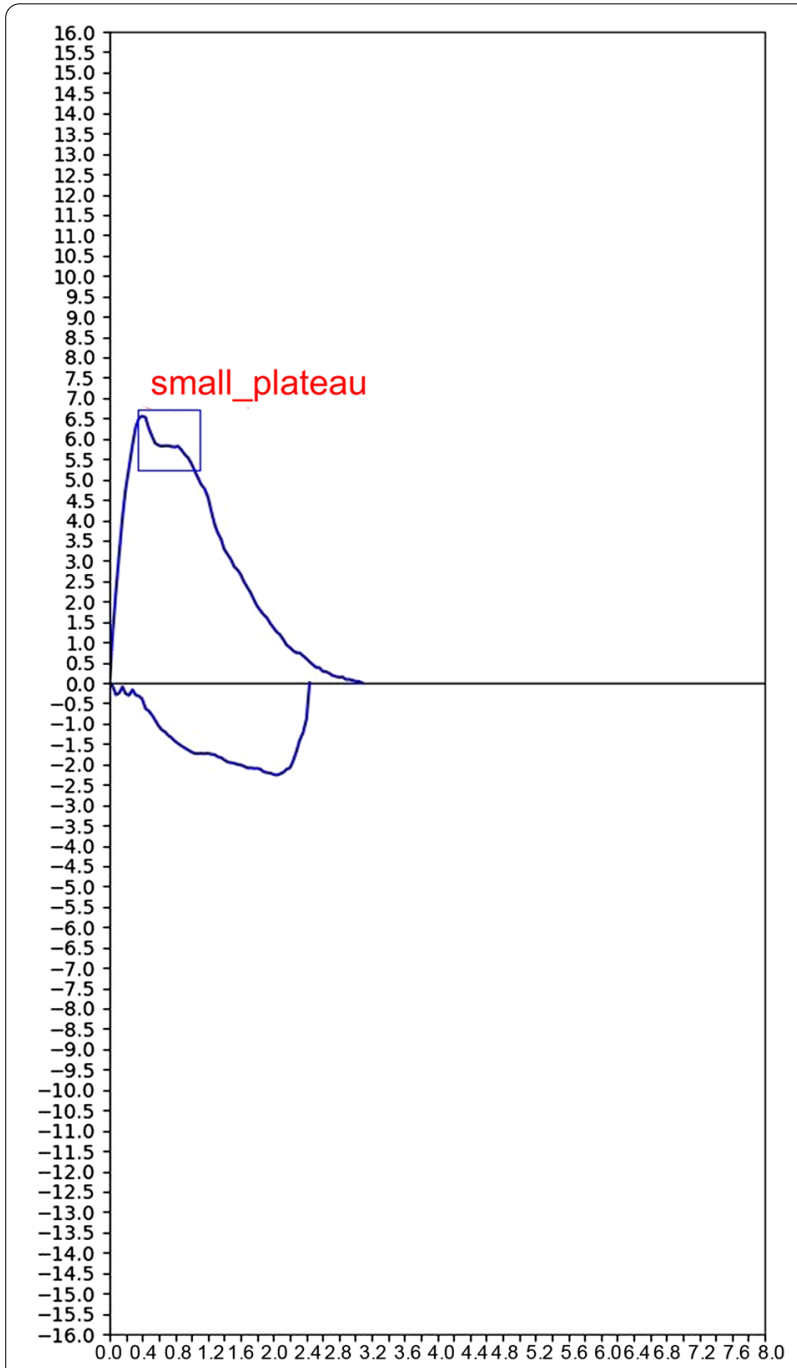

Fig. 7 Model output. The SP-Net drew a red bounding box to represent the detected position of the SP sign and also generated corresponding annotations. SP = small plateau; SP-Net $=$ SP-network

( $P=0.007$ and $P=0.003$, respectively). Patients with class 1 had a higher rate of PFT indication of COPD compared to class $4(P<0.0001)$ (Table 6$)$. In the height-stratified classes, there were 439 (24.6\%) with class 1, 759 (42.6\%) with class 2, 474 (26.6\%) with class 3, and 110 (6.2\%) subjects with class 4 . Compared with class 2 , class 4 were with higher median $\mathrm{FEV}_{1} \%$ predicted $(P=0.009)$. However, patients with class 1 had a higher $\mathrm{FEV}_{1} / \mathrm{FVC}$ ratio than other classes $(P<0.0001)$ (Table 7$)$.

\section{Laryngoscopy findings}

A total of 48 patients who underwent the laryngoscopy completed the FS tests. Of these, 32 (66.7\%) patients without SP sign and 16 (33.3\%) patients with the sign. Patients with SP sign were less likely to have laryngoscopy evidence of chronic pharyngitis or normal upper airway compared with those without the sign $(P=0.038)$ (Table 8). They showed a higher percentage of upper airway abnormalities. Figure 6 shows examples of these abnormalities. Further analysis of the degree of upper airway stenosis [24] due to these findings, we found that the degree of stenosis of patients with SP sign were all within $0 \sim 25 \%$, however, patients without SP sign showed different degrees of stenosis, which distributed in $~ 25 \%$, $50 \%$, 75\%, and $90 \%$ (Table 9 ).

\section{Automated recognition}

An example of the output of our model is shown in Fig. 7. After running the inference, our model would draw a red bounding box to represent the detected position of the SP sign and also generated corresponding annotations. Figure 8 visualizes two case studies consisting of laryngoscopy findings and corresponding model outputs. In both normal (A) and vocal cord polyp (B) cases, our SP-Net successfully detected the SP sign.

The performance of our proposed deep learning model was evaluated on the test set using five metrics. The evaluation showed that our model achieved 95.2\% accuracy, 93.7\% sensitivity, $95.7 \%$ specificity, $88.1 \%$ positive predictive value, and $97.8 \%$ negative predictive value (Table 10 and Fig. 9).

\section{Discussion}

Visual inspection of the flow-volume curve is a simple and valuable approach to diagnose and evaluate diseases, such as obstructive sleep apnea, upper airway obstruction, and unilateral main-stem bronchial obstruction [25-28]. The SP sign is a common but previously not widely recognized configuration of the curve that adds value to classify airway responsiveness. It has been defined as the presence of "a small plateau of the early phase of expiratory flow on the flow-volume curve" [5].

The current study found that patients with SP sign were mostly negative-BPTs. Since a positive-BPT usually means patient with AHR, when spirometry showing an SP sign, that might indicate the patient is less likely to have AHR. This result was in agreement with the 
a
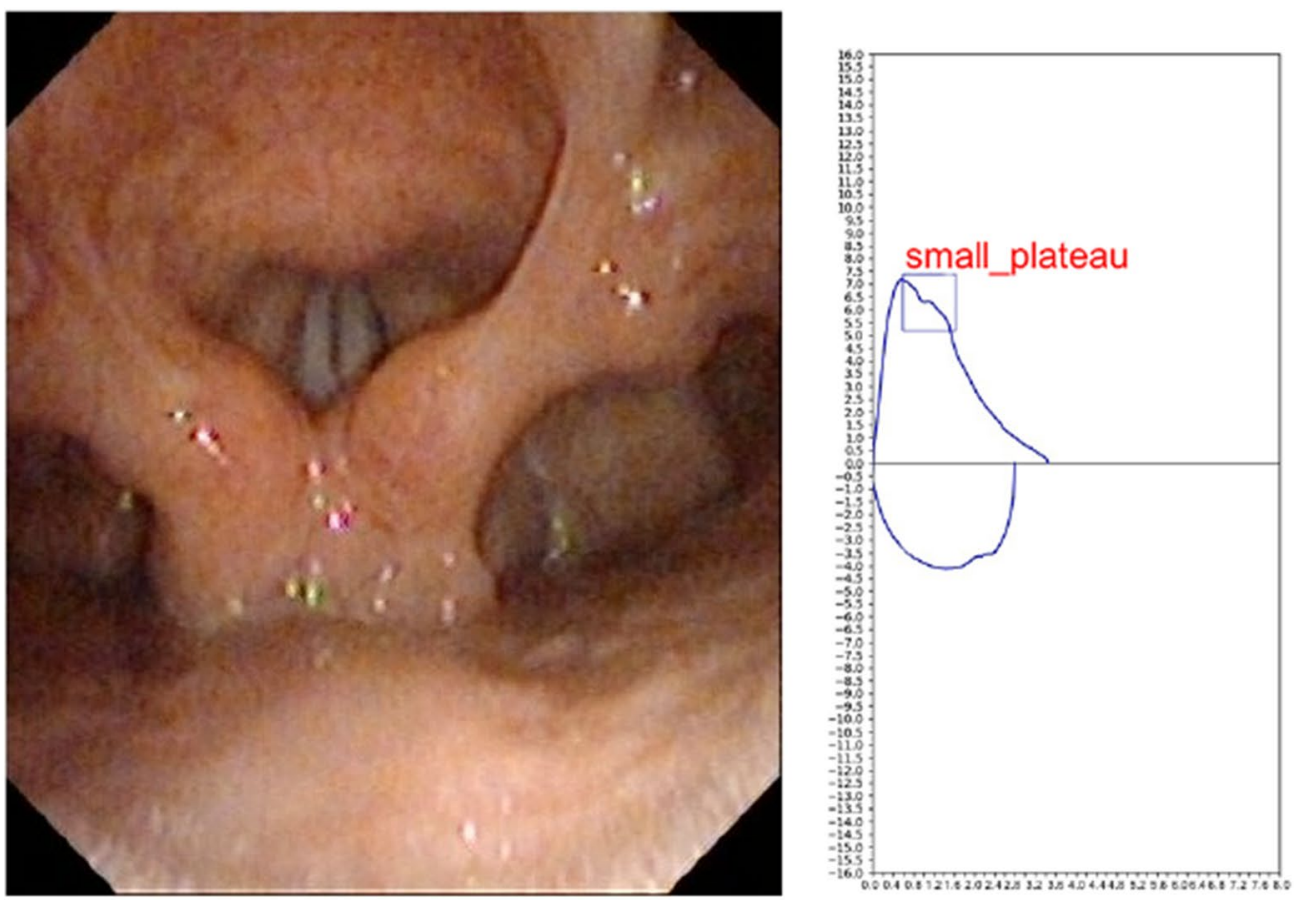

b
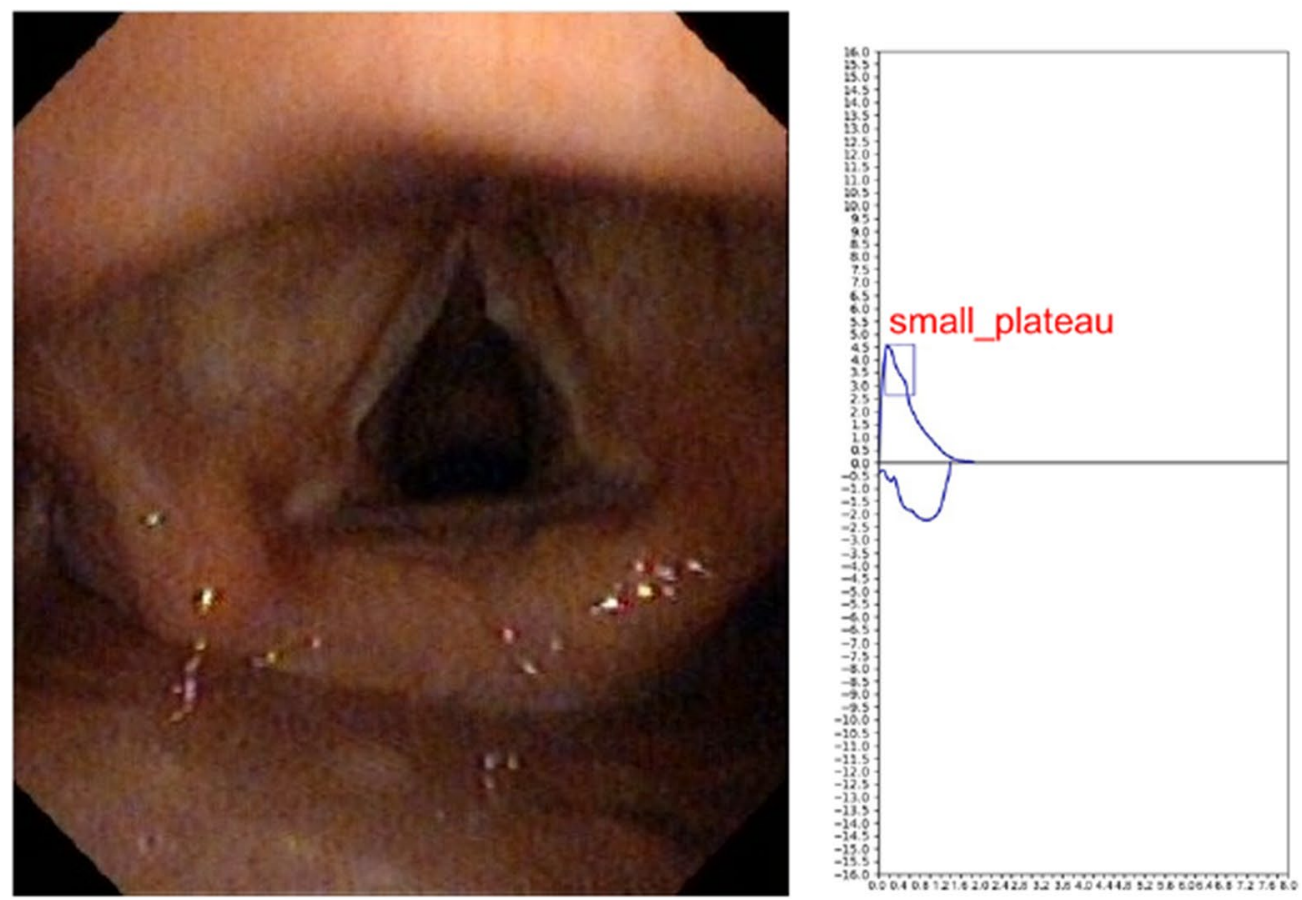

Fig. 8 Examples of the laryngoscopy findings and corresponding model outputs of the SP sign. a Normal; $\mathbf{b}$ vocal cord polyp. SP =small plateau

previous studies $[5,6]$. The prevalence of the SP sign in BDTs was only $2.7 \%$, of them, the rate of negative-BDTs was $88.6 \%$, this outcome indicated patients with SP sign had lower airway reversibility. Additionally, we noted the disappearing or narrowing of the width of the sign in the post-BPT maneuvers of patients with a positive-BPT, while the SP sign presented in the post-BDT maneuvers of patients with a positive-BDT. As indicated above, these findings supported the SP sign less on the flow-volume curves with severe ventilatory defects, or the sign could be masked because of the methacholine challengeinduced decline of PFT values. The median FVC, $\mathrm{FEV}_{1}$, 
Table 10 Performance evaluation ( $N=374$ records)

\begin{tabular}{llllll}
\hline & Accuracy & Sensitivity & Specificity & PPV & NPV \\
\hline SP-Net & $95.2 \%$ & $93.7 \%$ & $95.7 \%$ & $88.1 \%$ & $97.8 \%$ \\
& $(93.0-97.4)$ & $(91.2-96.2)$ & $(93.6-97.8)$ & $(84.8-91.4)$ & $(96.3-99.3)$ \\
\hline
\end{tabular}

Data are presented as mean and $95 \% \mathrm{Cl}$

SP, small plateau; SP-Net, SP-Network; PPV, positive predictive value; NPV, negative predictive value

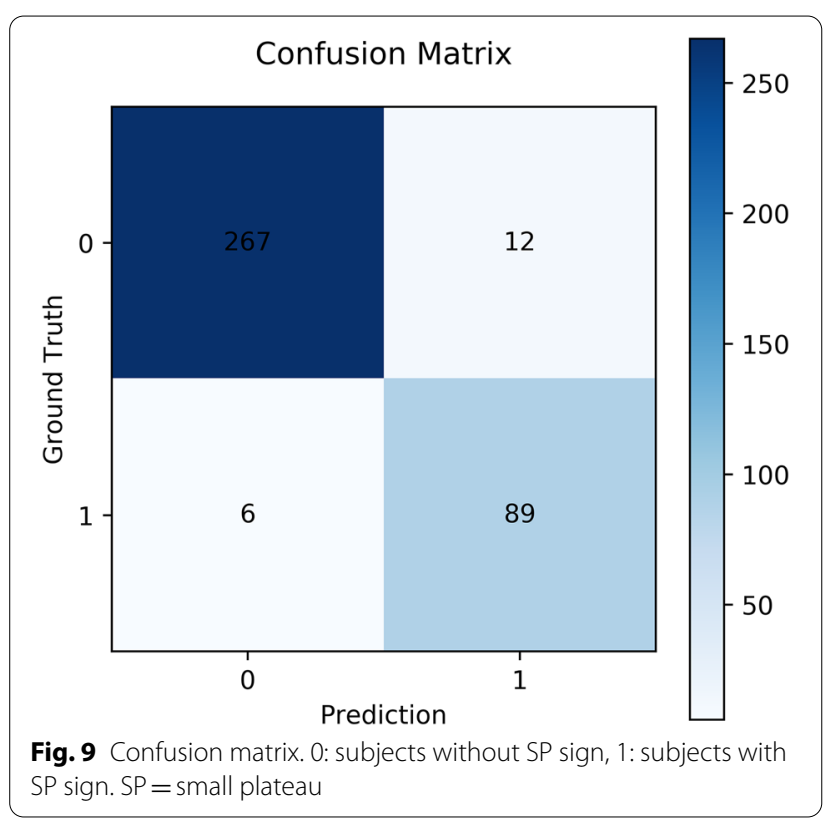

$\mathrm{FEF}_{50 \%}, \mathrm{FEF}_{75 \%}$, and MMEF \% predicted were all higher in patients with SP sign, whereas Li et al. [5] found no significant difference values between patients with or without SP sign using BPT data. In theory, our findings were also confirmed in BDT data. Moreover, a recent study has evaluated baseline spirometry variables as markers for AHR, they found that all baseline spirometry parameters were significantly lower in the positive AHR group. In their study, $\mathrm{FEF}_{50 \%}$ was proved to be a negative predictor for AHR [12]. In line with our study, SP sign also thought to be a negative marker, patients with SP sign presented higher $\mathrm{FEF}_{50 \%} \%$ predicted.

Further analysis of pre-BPTs questionnaires, we demonstrated that patients with SP sign were more likely to have PFT indications, including cough, rhinitis (sinusitis), and gastroesophageal reflux. On the contrary, the indications due to asthma and COPD were not significantly different compared to those without the sign. In theory, these diseases have been more often with symptoms like cough, sputum, and abnormal sensation of the throat, which were significantly associated with upper airway abnormalities.

The higher median $\mathrm{FEV}_{1} \%$ predicted and $\mathrm{FEV}_{1} / \mathrm{FVC}$ ratio in SP sign width-stratified class 4 compared to 1, demonstrated the sign presented more often in subjects with a normal-to-mild ventilatory defect. In addition, class 4 had lower rates of chronic cough and PFT indication of COPD, but had higher rates of PFT indications of bronchiectasis, chest pain, pleural effusion, and sleep disorder. These findings indicated that with the increase of the width of the sign, types of diseases were more complex and the symptoms were more diverse.

In 48 patients with laryngoscopy findings, we observed patients with SP sign had a lower prevalence of chronic pharyngitis or normal finding. They were more likely to have evidence of upper airway stenosis due to epiglottis cyst, hypertrophy of tonsils, vocal cord nodule, and throat tumor, etc. However, the degree of stenosis in patients with SP sign was mostly $0 \sim 25 \%$. In line with this tendency, Li et al. [5] observed the endoscopy findings of nine subjects with a negative-BPT and SP sign, they found that these subjects were excluded from severe central airway stenosis. These findings suggested that the SP sign may be an indicator to identify upper airway disorders.

Because the SP sign presented a good indicator to classify patients who were less likely to have AHR and the degree of severity of spirometric abnormality, we developed an SP-Net to automatically recognize it with an accuracy of $95.2 \%$. The confusion matrix has shown that the SP-Net was sensitive (93.7\%) when assessed on the test set $(\mathrm{N}=374$ cases). Six positive cases were wrongly detected negative because some curves have a concave shape at the same location of the SP sign. These shapes also appeared repeatedly in the different curves of one person. However, we did not know their physiological mechanism, fortunately, they were not common. A previous study proposed a neural network to detect upper airway obstruction caused by goiter using the flow-volume curve [29]. Artificial intelligence also helped with the interpretation and quality control of PFTs, together with the diagnosis of chronic respiratory diseases [14, 30, 31]. To the best of our knowledge, we were the first to use deep learning models for automatic detection of SP sign. Our object detection model not only could classify the image type, but could simultaneously locate the position of the SP sign. This additional feature could serve as interpretable guidance for the doctors to analyze and understand based on what visual cues the model classified a sample into the category of SP sign. It was very promising that our proposed deep learning algorithms could serve as a simple tool to aid SP sign recognition in primary care. In the future, the model can be integrated 
into the software of lung function equipment, more tasks of recognition of specific configuration of flowvolume curves, such as a saw-tooth sign, upper airway obstruction, and unilateral main bronchial stenosis will be developed. It will help alert the non-specialists to the potential presence of some disorders which sometimes be misdiagnosed.

A major limitation of this study was acquired data retrospectively, which could not observe symptoms of patients during BPTs. Although the SP sign was mostly present in patients with a negative-BPT, they were more likely to have evidence of symptoms due to upper airway abnormalities. The symptoms during BPTs will need to be observed in future work. Another drawback of our study was a lack of results of AHR in patients with laryngoscopy findings. They were only assigned to complete a baseline spirometry test, but not a BPT, since performing a BPT takes more time and may bring a bad experience to the subject. In addition, the level of inter-rater variability of authors when labelled the spirometry files could not be calculated. Because no one has re-labelled files that others' have already labelled. Finally, we only developed an approach to recognition SP sign using SPNet, future studies should incorporate clinical manifestations, PFT indices, and laryngoscopy findings to assist clinicians in the classification of healthy people, patients with asthma and COPD, as well as the prediction of airway responsiveness.

\section{Conclusions}

We analyzed the prevalence, clinical, and lung function characteristics of the SP sign. The sign mostly presented in patients with normal-to-mild ventilatory defect and a negative-BPT. Patients with SP sign were less likely to have AHR and severe ventilatory defects. We propose the SP sign as an indicator to perform the classification of patients with asthma who are mostly with AHR and patients with COPD who are mostly with obvious airway obstruction. The application of the sign is ideal for primary care that cannot carry out BPTs. Therefore, we developed SP-Net to automate the recognition of it. Additional studies will need to further define the sign, including its airway dynamics and physiology mechanisms.

\footnotetext{
Abbreviations

AHR: Airway hyperresponsiveness; BDT: Bronchodilator test; CV: Computer vision; BPT: Bronchoprovocation test; COPD: Chronic obstructive pulmonary disease; $\mathrm{FEF}_{\mathrm{x} \%}$ : Instantaneous forced expiratory flow when $\mathrm{x} \%$ of the FVC has been expired; FS: Forced spirometry; MMEF: Maximal mid-expiratory flow; PFT: Pulmonary function test; SP: Small plateau; SP-Net: SP-Network.
}

Acknowledgements

We thank Guangzhou Tianpeng Technology Co., Ltd for building the lung function testing and clinical information databases (Add: Zhujiang East Rd. \#11, Guangzhou 510627, China).

\section{Authors' contributions}

YW, WC, and CZ contributed to study design, hypothesis generation. LL, RH, and $J \mathrm{~L}$ contributed to the analysis and interpretation of the data. YG and JZ contributed to the charting review. YW, WC, and YL performed data analysis, manuscript drafting, and revising served as the primary author. JZ is the guarantor of this work and takes responsibility for the accuracy of the data analysis. All authors read and approved the final manuscript.

\section{Funding}

This work was supported by the National Key Technology R\&D Program (2018YFC1311901, 2016YFC1304603), the National Science \& Technology Pillar Program (2015BAl12B10), and the Science and Technology Program of Guangzhou, China (202007040003).

\section{Availability of data and materials}

The datasets used and/or analyzed during the current study are available from the corresponding author on reasonable request.

\section{Declarations}

\section{Ethics approval and consent to participate}

The study was approved by the Ethics Committee of the First Affiliated Hospital of Guangzhou Medical University (approval number: 2020124). Since it was an anonymized and retrospective research, written informed consent was waived.

\section{Consent for publication \\ Not applicable.}

\section{Competing interests}

The authors declare no conflicts of interest.

\section{Author details}

${ }^{1}$ National Center for Respiratory Medicine, State Key Laboratory of Respiratory Disease, National Clinical Research Center for Respiratory Disease, Guangzhou Institute of Respiratory Health, First Affiliated Hospital of Guangzhou Medical University, Yanjiang Road 151, Guangzhou 510120, Guangdong, People's Republic of China. ${ }^{2}$ Tsinghua-Berkeley Shenzhen Institute, Tsinghua University, Shenzhen 518055, People's Republic of China. ${ }^{3}$ Huawei Cloud BU El Innovation Laboratory, Huawei Technologies, Shenzhen 518129, People's Republic of China.

Received: 28 August 2021 Accepted: 3 November 2021

Published online: 09 November 2021

\section{References}

1. Miller MR, Hankinson J, Brusasco V, Burgos F, Casaburi R, Coates A, et al. Standardisation of spirometry. Eur Respir J. 2005:26(2):319-38.

2. Pellegrino R, Viegi G, Brusasco V, Crapo RO, Burgos F, Casaburi R, et al. Interpretative strategies for lung function tests. Eur Respir J. 2005;26(5):948-68.

3. Miller RD, Hyatt RE. Evaluation of obstructing lesions of the trachea and larynx by flow-volume loops. Am Rev Respir Dis. 1973;108(3):475-81.

4. Sanders MH, Martin RJ, Pennock BE, Rogers RM. The detection of sleep apnea in the awake patient. The "saw-tooth" sign. JAMA. 1981;245(23):2414-8.

5. Li M, Zheng J, Zhong N, Chen R, An J, Luo D. Preliminary study on the small-platform sign of flow-volume curve. Chin J Pract Intern Med. 1998;18(5):266-8 (in Chinese).

6. Xie Y, Liu C, Huang R, Xiuwen S, Zheng J. Clinical and characteristic parameters of small-platform of early phase of expiratory flow in forced expiratory flow-volume curve. Academ J GZh Med Univ. 2002;30(3):84-6 (in Chinese). 
7. Sterk PJ, Fabbri LM, Quanjer PH, Cockcroft DW, O'Byrne PM, Anderson SD, et al. Airway responsiveness. Standardized challenge testing with pharmacological, physical and sensitizing stimuli in adults. Report Working Party Standardization of Lung Function Tests, European Community for Steel and Coal. Eur Respir J Suppl. 1993;16:53-83.

8. Dharmage SC, Perret JL, Custovic A. Epidemiology of asthma in children and adults. Front Pediatr. 2019;7:246.

9. Suruki RY, Daugherty JB, Boudiaf N, Albers FC. The frequency of asthma exacerbations and healthcare utilization in patients with asthma from the UK and USA. BMC Pulm Med. 2017;17(1):74.

10. Gonzalez-Barcala FJ, Calvo-Alvarez U, Garcia-Sanz MT, Garcia-Couceiro N Martin-Lancharro P, Pose A, et al. Asthma exacerbations: risk factors for hospital readmissions. Ir J Med Sci. 2018;187(1):155-61.

11. Lim SY, Jo YJ, Chun EM. The correlation between the bronchial hyperresponsiveness to methacholine and asthma like symptoms by GINA questionnaires for the diagnosis of asthma. BMC Pulm Med. 2014;14:161.

12. Peled M, Ovadya D, Cohn J, Seluk L, Pullerits T, Segel MJ, et al. Baseline spirometry parameters as predictors of airway hyperreactivity in adults with suspected asthma. BMC Pulm Med. 2021;21(1):153.

13. He J, Baxter SL, Xu J, Xu J, Zhou X, Zhang K. The practical implementation of artificial intelligence technologies in medicine. Nat Med. 2019;25(1):30-6.

14. Das N, Verstraete K, Stanojevic S, Topalovic M, Aerts JM, Janssens W. Deeplearning algorithm helps to standardise ATS/ERS spirometric acceptability and usability criteria. Eur Respir J. 2020;56(6):2000603.

15. Krizhevsky A, Sutskever I, Hinton GE. Imagenet classification with deep convolutional neural networks. Adv Neural Inf Process Syst. 2012;25:1097-105.

16. Everingham M, Van Gool L, Williams CKI, Winn J, Zisserman A. The pascal visual object classes (VOC) challenge. Int J Comput Vision. 2010;88(2):303-38.

17. He K, Zhang X, Ren S, Sun J. Deep residual learning for image recognition. In: Proceedings of the IEEE conference on computer vision and pattern recognition. 2016. p. 770-8.

18. Ren S, He K, Girshick R, Sun J. Faster R-CNN: towards real-time object detection with region proposal networks. IEEE T Pattern Anal. 2017;39(6):1137-49.

19. Everingham M, Eslami SMA, Van Gool L, Williams CKI, Winn J, Zisserman A. The pascal visual object classes challenge: a retrospective. Int J Comput Vision. 2015;111(1):98-136.
20. Graham BL, Steenbruggen I, Miller MR, Barjaktarevic IZ, Cooper BG, Hall GL, et al. Standardization of spirometry 2019 update. An Official American Thoracic Society and European Respiratory Society Technical Statement. Am J Respir Crit Care Med. 2019;200(8):e70-88.

21. Coates AL, Wanger J, Cockcroft DW, Culver BH, Diamant Z, Gauvreau G, et al. ERS technical standard on bronchial challenge testing: general considerations and performance of methacholine challenge tests. Eur Respir J. 2017;49(5):1601526.

22. Chinese Thoracic Society. Guidelines for lung function examination part 2: spirometry. Chin J Tuberc Respir Dis. 2014;37(7):481-6 (in Chinese).

23. Girshick R. Fast R-CNN. In: 2015 IEEE international conference on computer vision (ICCV); 7-13 December 2015. 2015. p. 1440-8.

24. Freitag L, Ernst A, Unger M, Kovitz K, Marquette $\mathrm{CH}$. A proposed classification system of central airway stenosis. Eur Respir J. 2007;30(1):7-12.

25. Bourne MH Jr, Scanlon PD, Schroeder DR, Olson EJ. The sawtooth sign is predictive of obstructive sleep apnea. Sleep Breath. 2017;21(2):469-74.

26. Levent E, Sarıman N. Analysis of obstructive sleep apnea patients with "sawtooth sign" on the flow-volume curve. Sleep Breath. 2011;15(3):357-65.

27. Fiorelli A, Poggi C, Ardò NP, Messina G, Andreetti C, Venuta F, et al. Flowvolume curve analysis for predicting recurrence after endoscopic dilation of airway stenosis. Ann Thorac Surg. 2019;108(1):203-10.

28. Ko Y, Yoo JG, Yi CA, Lee KS, Jeon K, Um SW, et al. Changes in the flow-volume curve according to the degree of stenosis in patients with unilateral main bronchial stenosis. Clin Exp Otorhinolaryngol. 2015;8(2):161-6.

29. Bright P, Miller MR, Franklyn JA, Sheppard MC. The use of a neural network to detect upper airway obstruction caused by goiter. Am J Respir Crit Care Med. 1998;157(6 Pt 1):1885-91.

30. Topalovic M, Das N, Burgel PR, Daenen M, Derom E, Haenebalcke C, et al. Artificial intelligence outperforms pulmonologists in the interpretation of pulmonary function tests. Eur Respir J. 2019;53(4):1801.

31. Spathis D, Vlamos P. Diagnosing asthma and chronic obstructive pulmonary disease with machine learning. Health Informatics J. 2019;25(3):811-27.

\section{Publisher's Note}

Springer Nature remains neutral with regard to jurisdictional claims in published maps and institutional affiliations.
Ready to submit your research? Choose BMC and benefit from:

- fast, convenient online submission

- thorough peer review by experienced researchers in your field

- rapid publication on acceptance

- support for research data, including large and complex data types

- gold Open Access which fosters wider collaboration and increased citations

- maximum visibility for your research: over $100 \mathrm{M}$ website views per year

At BMC, research is always in progress.

Learn more biomedcentral.com/submissions 